\title{
Philosophiques
}

\section{Le « Wittgenstein intermédiaire » et les mathématiques modernes}

\section{Sören Stenlund et Anne-Marie Boisvert}

Volume 39, numéro 1, printemps 2012

La période intermédiaire de Wittgenstein

URI : https://id.erudit.org/iderudit/1011613ar

DOI : https://doi.org/10.7202/1011613ar

Aller au sommaire du numéro

Éditeur(s)

Société de philosophie du Québec

ISSN

0316-2923 (imprimé)

1492-1391 (numérique)

Découvrir la revue

Citer cet article

Stenlund, S. \& Boisvert, A.-M. (2012). Le « Wittgenstein intermédiaire » et les mathématiques modernes. Philosophiques, 39(1), 125-161.

https://doi.org/10.7202/1011613ar
Résumé de l'article

Dans cet article, j'essaie de montrer que le dépassement et le rejet du dogmatisme sont un aspect décisif du changement dans la pensée de Wittgenstein qui a eu lieu au début des années 30 , quand il commence à mettre en valeur l'autonomie de la grammaire du langage et à parler d'images grammaticales et de jeux de langage en tant qu'objets de comparaison. En examinant certains traits fondamentaux de ce changement, je mettrai en évidence l'impulsion et les idées décisives que Wittgenstein a reçues des nouveaux développements dans les mathématiques et la science naturelle du début du $20^{\mathrm{e}}$ siècle. 


\title{
Le «Wittgenstein intermédiaire» et les mathématiques modernes
}

\author{
SÖREN STENLUND \\ Université de Uppsala, Suède \\ soren.stenlund@filosofi.uu.se
}

\begin{abstract}
RÉSUMÉ. - Dans cet article, j'essaie de montrer que le dépassement et le rejet du dogmatisme sont un aspect décisif du changement dans la pensée de Wittgenstein qui a eu lieu au début des années 30 , quand il commence à mettre en valeur l'autonomie de la grammaire du langage et à parler d'images grammaticales et de jeux de langage en tant qu'objets de comparaison. En examinant certains traits fondamentaux de ce changement, je mettrai en évidence l'impulsion et les idées décisives que Wittgenstein a reçues des nouveaux développements dans les mathématiques et la science naturelle du début $d u 20^{e}$ siècle.
\end{abstract}

\begin{abstract}
It will be argued that the overcoming and avoiding of dogmatism is a decisive feature of the change in Wittgenstein's thinking that takes place in the beginning of the 1930's when he starts to emphasise the autonomy of the grammar of language and to talk about grammatical pictures and language games as objects of comparison. By examining certain crucial features in this change in Wittgenstein's thinking, it will be shown that he received decisive impulses and ideas from new developments in mathematics and natural science in the early $20^{\text {th }}$ century.
\end{abstract}

\section{Introduction}

Le travail de Wittgenstein en philosophie des mathématiques occupe une place beaucoup plus centrale dans sa réflexion d'ensemble et dans la continuité de sa pensée philosophique que celle qui lui est généralement reconnue dans la conception dite "des deux Wittgenstein» (laquelle introduit une distinction entre le premier Wittgenstein [l'auteur du Tractatus] et le deuxième Wittgenstein [l'auteur des Recherches philosophiques]). Pour mettre cela en lumière, il nous faut examiner plus attentivement le travail de Wittgenstein au cours de la "période intermédiaire» (en gros, de I929 à I936) ${ }^{1}$. Je me concentrerai dans le présent article sur le changement qui s'est opéré dans la pensée de Wittgenstein principalement au début des années trente. À travers l'examen des aspects essentiels de ce changement, je chercherai à montrer l'influence décisive que les nouveaux développements en mathématiques et en sciences naturelles ont eue sur Wittgenstein à cette époque. Il en a tiré des idées qui ont eu une influence, non seulement sur sa conception des mathématiques, mais également sur sa conception du langage et de la nature de la philosophie en général.

1. Gerrard (I99I) a souligné l'importance de la "période intermédiaire » pour la compréhension de la philosophie des mathématiques de Wittgenstein. 
Un aspect de la philosophie mathématique de Wittgenstein est présent du début à la fin: c'est son insistance sur la différence entre les propositions mathématiques et les propositions de l'expérience. Selon Wittgenstein, les propositions mathématiques — purement mathématiques — n'ont pas de contenu descriptif; elles ne "portent sur rien ", au sens où les propositions de la physique ou de la biologie portent sur quelque chose ${ }^{2}$. La différence ne tient pas seulement au fait que la physique ou la biologie ont pour objet des phénomènes empiriques observables, alors que ce n'est pas le cas des mathématiques. C'est plutôt que les propositions mathématiques ne sont pas des propositions, si le fait de porter sur quelque chose de donné à l'avance est considéré comme une caractéristique essentielle des propositions. Ainsi, les propositions du Tractatus, par exemple, ne comportent pas de propositions mathématiques.

Or, selon une conception fort courante, il existe un royaume d'entités sur lesquelles portent les propositions mathématiques et que ces dernières décrivent. Dans une conférence prononcée en 1939, Wittgenstein a exposé la source de ce qui lui paraît problématique dans cette conception comme suit:

La difficulté que l'on rencontre quand on considère les mathématiques comme nous le faisons est de créer une section particulière - de séparer la mathématique pure de son application. Il est particulièrement difficile de savoir où faire passer cette ligne de séparation. Se sont en effet développées certaines branches des mathématiques dont le charme tient à ce qu'en elles les mathématiques pures ont l'apparence de mathématiques appliquées - appliquées à ellesmêmes. Ainsi est née toute cette affaire du royaume mathématique ${ }^{3}$.

Au sens où Wittgenstein utilise ici les mots «applique» et "application ", un système mathématique appliqué est toujours appliqué à quelque chose qui existe à l'avance à l'extérieur du système qui est appliqué. Celui-ci est appliqué à un objet qui existe indépendamment du système mathématique, comme lorsque nous disons que le système de la géométrie euclidienne pure est appliqué en astronomie afin de décrire les mouvements des planètes. Les planètes et leurs mouvements en tant que phénomènes empiriques existent à l'avance, indépendamment de la géométrie et de son application. Quand une notion mathématique est appliquée à l'intérieur des mathématiques, et que ce sens d'application est maintenu (ce à quoi la «prose» des mathématiques nous invite), il semble qu'il y ait un royaume mathématiqueexistant à l'avance. Par exemple, lorsque nous parlons du nombre des

2. Dans les Remarques philosophiques, $\mathbb{1} 57$, p. I78, on trouve le commentaire suivant qui met en lumière cet aspect du symbolisme mathématique: "Souvenons-nous qu'en mathématique les signes mêmes font la mathématique, et non décrivent la mathématique. [...] On ne peut pas écrire la mathématique [comme par exemple on peut écrire l'histoire], on peut seulement la faire.»

3. Cours sur les fondements des mathématiques. Cambridge 1939, Cours XV, p. I 50. 
racines d'une équation, ce nombre paraît exister pour être découvert dans un royaume à l'extérieur du système arithmético-algébrique au sein duquel la notion de racine d'une équation est inventée et caractérisée.

Séparer un système de mathématiques pures de ses applications, au sens décrit dans la citation ci-dessus, revient à présenter le système en question comme un système conceptuellement autonome. Cette idée d'autonomie et son influence sur la conception wittgensteinienne de la grammaire à partir des années trente constituent le thème principal du présent article. L'idée d'autonomie est en liaison avec certains courants importants en mathématiques modernes et en sciences naturelles du début du vingtième siècle, par exemple avec les travaux de Heinrich Hertz en mécanique et de David Hilbert en géométrie, et plus généralement avec la méthode axiomatique de Hilbert.

Par "mathématiques modernes", nous entendrons dans cet article les nouveaux courants en mathématiques qui se sont fait jour à la fin du dixneuvième siècle et au début du vingtième siècle, alors que l'ancienne conception des mathématiques comme science des quantités est perçue comme dépassée et inadéquate eu égard au changement et au développement qui s'opèrent à l'intérieur de la discipline des mathématiques, en particulier eu égard à l'insistance désormais mise sur la rigueur formelle et sur les mathématiques pures comme discipline indépendante et autonome ${ }^{4}$.

\section{Le problème du dogmatisme et l'autonomie de la grammaire}

L'idée d'autonomie a inspiré à Wittgenstein une façon de surmonter le dogmatisme dont il s'était, selon lui, rendu coupable dans le Tractatus. Il décrit par exemple les caractéristiques des mathématiques modernes sur lesquelles il focalise alors son attention comme suit:

[L]es constructions arithmétiques sont autonomes, comme les constructions géométriques, et par là, pour ainsi dire, garantissent elles-mêmes leur applicabilité ${ }^{5}$ et l'application du calcul doit prendre soin d'elle-même. Et c'est ce qui est correct dans le formalisme ${ }^{6}$.

Un trait récurrent dans la philosophie de Wittgenstein est l'usage fréquent de problèmes spécifiques à un domaine, en tant qu'analogies, afin

4. Voir Mehrtens (1990), Epple (2003), et Gray (2006). Mehrtens se concentre en particulier sur la crise fondationnelle comme symptôme de ce changement, laquelle a rendu nécessaire la révision et la redéfinition de l'identité des mathématiques en tant que discipline. Il présente Hilbert comme le principal défenseur des mathématiques modernes, et comme le principal défenseur de l'autonomie des mathématiques et du discours mathématique. Au début des années trente, Wittgenstein (contrairement à la plupart des mathématiciens et logiciens) a visiblement été sensible à cet aspect culturel des mathématiques et conscient du fait que cette évolution scientifique faisait partie, dans une certaine mesure, d'un changement culturel plus large.

5. Remarques philosophiques, $\mathbb{S}$ I I I, p. I27.

6. Remarques sur les fondements des mathématiques, troisième partie, $\mathbb{4}$, p. I39. 
d'illustrer certains points dans d'autres domaines. Nous retrouvons par exemple ce trait dans une conférence donnée au printemps I93 I, au cours de laquelle Wittgenstein affirme que «la relation entre l'attente et le remplissement est très exactement celle de l'opération et du résultat ${ }^{7}{ }$. Et contrairement à ce à quoi l'on pourrait s'attendre, étant donné l'image du second Wittgenstein telle que véhiculée par la "conception des deux Wittgenstein ", l'usage d'analogies tirées des mathématiques pour illustrer des points dans d'autres domaines se fait plus abondant dans son œuvre à partir du début des années trente.

L'idée de concevoir le langage, ou la structure logique du langage, comme un calcul, est une idée qui est déjà centrale dans le Tractatus. L'idée de comparer le langage ordinaire ou certaines de ses parties à des jeux, et par suite l'idée de jeux de langage, a été inspirée à Wittgenstein par l'usage que les mathématiciens formalistes ont fait du jeu d'échecs pour mettre en lumière certains traits conceptuellement signifiants des mathématiques. Wittgenstein a certainement lu le passage suivant du mathématicien Johannes Thomae (cité quasiment en entier par Frege dans Les lois fondamentales de l'arithmétique (Grundgesetze der Arithmetik):

La conception formelle des nombres s'impose des limites plus modestes que la conception logique. Elle ne demande pas ce que sont et ce que doivent être les nombres, mais plutôt ce que l'on exige d'eux en arithmétique. Dans la conception formelle, l'arithmétique est un jeu avec des signes qu'on peut décrire comme vides, c'est-à-dire qu'ils n'ont (dans le jeu du calcul) pas d'autre contenu que celui qui leur a été attribué et qui a trait à leur comportement à l'égard de certaines règles de combinaison (règles du jeu). De même, un joueur d'échecs utilise ses pièces, il leur attribue certaines propriétés qui conditionnent leur comportement dans le jeu, et les pièces elles-mêmes ne sont que des signes extérieurs de ce comportement. Certes, il y a une différence importante entre le jeu d'échecs et l'arithmétique. Les règles du jeu d'échecs sont arbitraires, alors que le système de règles en arithmétique est tel que les nombres peuvent être rattachés, par le biais de simples axiomes, à diverses intuitions, de sorte qu'ils sont d'une utilité essentielle dans la connaissance de la nature. - Le point de vue formel nous délivre de toutes les difficultés métaphysiques, c'est le principal avantage qu'il nous offre ${ }^{8}$.

L'analogie du jeu d'échecs est utilisée fréquemment dans les écrits de Wittgenstein de la période intermédiaire, en particulier dans ses conférences des années trente dans lesquelles celui-ci explore ledit point de vue formel et l'étend au langage en général. L'analogie du jeu d'échecs est également présente dans ses écrits philosophiques ultérieurs, notamment dans les Recherches. Quand Wittgenstein exprime un certain regret que Frege n'ap-

7. Les Cours de Cambridge 1930-1932, p. 7I.

8. Citation tirée de Epple 2003, p. 30I (NDLT: ma traduction, d'après la version anglaise). 
précie pas l'utilité de la conception formaliste des mathématiques, ce qu'il semble avoir en tête, c'est l'usage que les formalistes font de l'analogie du jeu d'échecs pour s'affranchir de l'image biaisée en faveur du modèle «nomobjet» des signes du langage en mathématiques.

Dans la controverse opposant Hilbert et Frege sur la question de savoir si les axiomes d'un système mathématique doivent demeurer tributaires du sens des mots qui les composent (comme le soutenait Frege), ou si les axiomes peuvent être considérés comme définissant ou constituant le sens des mots (un trait essentiel de la méthode axiomatique de Hilbert), Wittgenstein se situait à l'évidence davantage du côté de Hilbert'9

Dans les Remarques philosophiques, Wittgenstein nous invite à considérer une équation mathématique comme une règle de syntaxe, pour ensuite remarquer que cela

rend également compréhensibles les essais des formalistes qui voient dans la mathématique un jeu avec les signes.

Des règles des signes — par exemple les définitions — peuvent sans doute être saisies comme des propositions qui traitent de signes, mais il n'est pas indispensable du tout de les saisir comme des propositions. Elles sont les moyens auxiliaires du langage. Des moyens auxiliaires d'un autre type que les propositions du langage ${ }^{10}$.

Wittgenstein tend ici à glisser d'une remarque à propos des signes du langage en mathématiques au langage en général. Et ce glissement, dans une de ses conférences prononcées au début des années trente, est conscient et explicite:

Il est utile de parler des échecs, qui sont semblables aux mathématiques, mais qui, à la différence des mathématiques, ont l'avantage de n'avoir aucune aura ${ }^{11}$.

Nous pouvons comparer l'emploi du langage au fait de jouer un jeu en accord avec des règles exactes, parce que tous les troubles philosophiques proviennent de ce que nous simplifions extrêmement un système de règles ${ }^{12}$.

Le mot «comparer» est important ici. Wittgenstein ne dit pas qu'il fait ces comparaisons parce qu'il pense que faire des mathématiques ou utiliser un langage soit réellement jouer un jeu avec des règles précises, ou que le langage soit effectivement composé de jeux dotés de règles précises. Ce que Wittgenstein vise ici n'est pas une doctrine sur la nature du langage, mais une méthode d'investigation conceptuelle. Dans la Grammaire philosophique, il explique que:

9. Voir l'objection de Wittgenstein à la critique frégéenne des formalistes dans la Grammaire philosophique, première partie, I, $\mathbb{2}$ 2, p. 60.

10. Remarques philosophiques, $\mathbb{1}$ I I , p. I38.

11. Les cours de Cambridge 1932-1935, p. 6I.

12. Les cours de Cambridge 1932-1935, p. 65-66. 
Appeler l'arithmétique un jeu est tout aussi faux qu'appeler jeu le fait de pousser des pièces d'échecs (en suivant les règles de ce jeu), car cela peut aussi être un calcul.

[...devrait-on] dire: non, le mot arithmétique n'est pas le nom d'un jeu. (Naturellement, il s'agit là encore d'une trivialité). Mais on peut expliquer la signification du mot "Arithmétique" par la relation de l'arithmétique à un jeu arithmétique et par la relation du problème d'échecs au jeu d'échecs ${ }^{13}$.

Waismann a exposé clairement dans le passage qui suit la manière dont Wittgenstein conçoit le fonctionnement de la méthode consistant à comparer l'usage du langage à un jeu joué selon des règles exactes:

Frege critique l'idée selon laquelle l'arithmétique est un simple jeu de signes. Maintenant il y a dans cette critique quelque chose d'inexact, et il serait bien séduisant de poursuivre cet examen. Mais nous pourrions aussi adopter un tout autre point de vue. Nous pourrions dire: laissons tout à fait de côté la question de savoir si l'arithmétique est un jeu ou non. Une chose est claire: il doit y avoir ici une certaine parenté, car sinon personne ne serait arrivé à cette idée. Examinons donc une bonne fois le jeu! Plaçons l'examen du jeu à côté de celui de l'arithmétique et laissons l'un jeter une lumière sur l'autre! Soyons tout à fait justes, n'affirmons rien, et laissons au contraire les choses parler d'elles-mêmes!

Tel est le point de vue duquel nous voulons considérer le langage. Nous ne voulons pas dogmatiser mais laisser le langage être tel qu'il est et le mettre en parallèle avec une image grammaticale dont nous maîtrisons complètement les propriétés. Nous construisons pour ainsi dire un cas idéal mais sans la prétention qu'il s'accorde avec quoi que ce soit. Mais nous le construisons seulement en vue d'obtenir un schéma synoptique avec lequel comparer le langage, quelque chose comme un aspect qui n'affirme rien encore et qui n'est pas non plus faux ${ }^{14}$.

Remarquons dans cette citation que c'est le souci d'éviter le dogmatisme qui constitue la caractéristique la plus importante de ce "point de vue entièrement différent » dans lequel les images grammaticales et les jeux sont

13. Grammaire philosophique, deuxième partie, III, II, p. 375.

Wittgenstein a fait essentiellement la même remarque sous une forme plus concise au cours d'une de ses conférences à Cambridge en I939, en soulignant d'emblée la chose suivante: "On a souvent dit des mathématiques qu'elles étaient un jeu, comparable au jeu d'échecs. En un sens, cette affirmation est manifestement fausse - car les mathématiques ne sont pas un jeu au sens ordinaire. En un sens, elle est manifestement vraie - une certaine similitude existe en effet. Il ne faut pas éluder la question, mais l'explorer. Comparer les mathématiques à un jeu est parfois utile, mais prête parfois à confusion" (Cours sur les fondements des mathématiques. Cambridge 1939, Cours XV, p. I42, l'italique est de Stenlund).

14. G. Baker (dir.) 2003, p. 279. (Traduction française tirée de Dictées de Wittgenstein à Waismann et pour Schlick, p. I40.) La citation est tirée d'un manuscrit datant du début des années trente qui était censé paraître sous la forme d'une publication conjointe de Waismann et Wittgenstein, mais qui n'a jamais été complété. Même s'il s'agit d'une transcription de Waismann, la voix qu'on y entend est sans conteste celle de Wittgenstein. 
utilisés comme des objets de comparaison. Wittgenstein a fait en 1937 la remarque suivante:

La seule façon, veux-je dire, dont nous puissions éviter que nos prétentions ne soient injustifiées, ou vides, consiste à regarder l'idéal, dans notre activité théorique, pour ce qu'il est, c'est-à-dire comme un objet de comparaison pour ainsi dire comme étalon -, au lieu d'en faire un préjugé auquel tout doit se conformer. C'est en cela en effet que consiste le dogmatisme, dans lequel la philosophie tombe si aisément ${ }^{15}$.

Mais Wittgenstein avait déjà fait essentiellement la même remarque, en plus détaillée, au mois d'août I93I, dans le cadre d'une comparaison entre son approche et celle d'Oswald Spengler en histoire des cultures:

Spengler serait mieux compris s'il disait: Je compare différentes périodes culturelles à la vie de plusieurs familles; à l'intérieur d'une famille il y a un air de famille, tandis qu'il y a aussi une ressemblance entre des membres de familles différentes; mais l'air de famille se distingue de cette autre ressemblance par tel et tel caractère, etc. Je veux dire: l'objet de comparaison, l'objet dont est tirée la façon de voir les choses, doit nous être indiqué, afin d'éviter que des inexactitudes ne se glissent sans cesse dans la discussion. Faute de quoi tout ce qui vaut pour le paradigme de la théorie, on le prétendra valable volens nolens également pour l'objet dont on fait la théorie, et l'on prétendra qu' «il doit toujours... »

Or cela vient de ce que l'on cherche à s'appuyer dans la théorie elle-même sur les caractéristiques du paradigme. Mais du fait que l'on mélange paradigme et objet, on se voit contraint d'attribuer dogmatiquement à l'objet ce qui ne doit caractériser que le paradigme. On croit par ailleurs que la théorie n'aurait pas l'universalité qu'on veut lui donner si elle ne s'accordait vraiment qu'avec le premier cas. Mais le paradigme doit justement être proposé comme tel; c'est-àdire comme caractérisant toute la théorie, dont il détermine la forme. Il est par conséquent au sommet, et s'il est universel, c'est parce qu'il détermine la forme de la théorie, et non parce que tout ce qui vaut pour lui, et pour lui seul, devrait être énoncé de tous les objets de la théorie.

On devrait donc poser à toutes les prétentions hyperboliques, dogmatisantes, la question suivante: qu'y a-t-il donc là d'effectivement vrai ? Ou encore: dans quel cas cela est-il donc effectivement valable ${ }^{16}$ ?

Ce qui rend possible de considérer les jeux de langage et les images grammaticales uniquement comme des objets de comparaison, c'est l'idée d'autonomie de la grammaire du langage. C'est sur la base de l'autonomie de la grammaire que Wittgenstein peut affirmer qu' "il ne s'agit pas ici d'une relation à la réalité qui maintiendrait la grammaire dans son ornière!». La "relation du langage à la réalité » au moyen des définitions ostensives ne

15. Remarques mêlées, p. 37 .

16. Ibid., p. 24-25. 
rend pas la grammaire contraignante (ne la justifie pas). [...] «La relation à la réalité ne fait que développer le langage, elle ne l'engage à rien ${ }^{17}$. »

L'idée de l'autonomie de la grammaire forme la base de la méthode de construction de nouvelles notations, ou de nouveaux jeux de langage (souvent purement fictifs) en tant qu'objets de comparaison, pour observer, par exemple, jusqu'où peut aller telle analogie entre deux usages d'une expression. C'est la raison pour laquelle l'idée d'autonomie m'apparaît si importante eu égard au changement qui s'est opéré dans la pensée de Wittgenstein au tout début des années trente.

Wittgenstein pense à cette époque à la grammaire comme à un calcul doté d'un ensemble fixe de règles, et il exprime l'idée d'autonomie comme suit :

La grammaire n'est redevable d'aucune réalité. Les règles grammaticales ne font que déterminer la signification (la constituer), de ce fait elles ne sont pas responsables de la signification et, dans cette mesure, sont arbitraires $^{18}$.

Les règles de la grammaire sont arbitraires au même sens que le choix d'une unité de mesure. Mais cela peut simplement signifier que cette unité de mesure est indépendante de la longueur de l'objet de la mesure; et non pas que le choix d'une unité est "vrai», le choix d'une autre unité "faux», comme la donnée de longueur est vraie ou fausse ${ }^{19}$.

L'affirmation de Wittgenstein selon laquelle les règles de grammaire sont en un sens arbitraire est importante, étant donné qu'il y a sûrement des sens du mot «arbitraire » où le choix d'une unité de mesure dans un certain contexte n'est pas arbitraire. Il peut y avoir de bonnes raisons pratiques d'utiliser une unité plutôt qu'une autre dans une situation donnée. Il en est de même, à certains égards, du langage humain, qui n'est pas utilisé de manière autonome, mais est conditionné par des facteurs causaux tels que la constitution de l'organisme humain, les organes des sens, le comportement ordinaire des êtres humains, certains faits de nature généraux, etc. Si le langage est un phénomène de la vie humaine ${ }^{20}$, il ne peut pas ne pas être conditionné par la nature des êtres humains, par la nature du monde dans lequel nous vivons et par les traditions et les formes de vie au sein desquelles les pratiques linguistiques ont évolué. Ces circonstances contraignent, mais ne déterminent pas ou ne justifient pas nos concepts ou nos règles de grammaire ${ }^{21}$.

17. Grammaire philosophique, deuxième partie, III, I 5, p. $40 \mathrm{O}$.

18. Grammaire philosophique, première partie, $\mathrm{X}, \mathbb{I}$ I33, p. 240.

19. Ibid., p. 242.

20. Remarques sur les fondements des mathématiques, sixième partie, $\mathbb{S} 47, \mathrm{p} .285$.

21. Voir Medina (2004). Les aspects non arbitraires de l'usage du langage ont acquis davantage d'importance pour Wittgenstein vers le début des années quarante et plus tard alors qu'il aborde le langage dans une perspective plus anthropologique. Mais cela ne veut pas dire 
On a dit parfois que la conception du langage comme calcul avec des règles fixes qui est celle de Wittgenstein au début des années trente est une survivance du Tractatus (la seule différence étant que le calcul en question n'est plus limité au seul calcul des fonctions de vérité, comme dans le Tractatus). Mais c'est ne pas voir l'essence du changement qui s'est opéré au tout début des années trente, à savoir le rejet du dogmatisme qui entachait la conception du calcul dans le Tractatus, comme l'explique Wittgenstein dans sa comparaison entre sa conception et celle de Spengler. Lorsque Wittgenstein fait la remarque suivante dans le Grand Cahier: "Je considère le langage et la grammaire comme un calcul, c'est-à-dire comme un processus qui suit des règles fixes ${ }^{22}$ ", il ne donne pas de réponse à la grande question philosophique de la nature et de la structure logique du langage en général. Cette remarque (et d'autres similaires) a plutôt un caractère méthodologique: Wittgenstein élucide et clarifie son approche du langage par rapport à d'autres approches (par exemple, la théorie causale de la signification de Russell dans Analyse de l'esprit). Il précise son "objet de comparaison, l'objet dont est tirée la façon de voir les choses ", afin d'éviter de se voir "contraint d'attribuer dogmatiquement à l'objet ce qui ne doit caractériser que le paradigme».

Si Wittgenstein adhérait encore à une conception du calcul dogmatique, semblable de ce point de vue à celle du Tractatus, il n'aurait certainement pas affirmé la chose suivante: "Nous pouvons dire: considérons le langage sous le rapport de ses règles. Si, ici et là, il n'y a pas de règles, alors c'est là le résultat de notre investigation. »Et c'est pourtant ce qu'il affirme dans le Grand Cahier23.

Wittgenstein énonce clairement le statut qu'il attribue au début des années trente à la conception du calcul dans Le cahier bleu:

Quand nous parlons du langage comme d'une symbolisation qui se conforme à des règles, on peut découvrir ce que nous entendons par là par référence à la science et aux mathématiques, mais il est assez rare que dans son usage ordinaire le langage concorde avec ces modèles d'exacte précision. Alors pourquoi, dans ces recherches, confrontons-nous toujours l'usage des mots avec un usage qui se conformerait à des règles strictes? La réponse ne serait-elle pas que nous essayons ainsi de résoudre des énigmes qui proviennent justement de notre façon de considérer le langage ${ }^{24}$ ?

qu'il récuse le sens selon lequel la grammaire est autonome, comme le montre clairement entre autres la remarque RP II, xii (Recherches philosophiques, p. 32I).

22. The Big Typescript, TS 213, p. 203. (NDLT: ouvrage non traduit en français. Ma traduction, d'après la version anglaise.)

23. The Big Typescript, TS 213, p. 199 (NDLT: ouvrage non traduit en français. Ma traduction, d'après la version anglaise.)

24. Le cahier bleu, p. 79 . 
En outre, on voit clairement ici que les sources principales d'inspiration de l'approche wittgensteinienne sont la science et les mathématiques - non pas cependant au sens où Russell, Carnap et les positivistes logiques se sont également inspirés des méthodes des sciences et des mathématiques dans le développement de leurs théories du langage. On pourrait dire que ces derniers ont adopté les méthodes exactes des sciences et des mathématiques comme voies vers la vérité au sujet du langage, alors que pour Wittgenstein ces méthodes sont des voies vers la source de problèmes et d'énigmes. Et il ne suffit pas de dire simplement que les énigmes naissent de cette façon de considérer le langage: il nous faut suivre la voie pour de bon pour nous en rendre compte.

Cette façon de concevoir l'usage du langage comme une opération de calcul avec des règles fixes posait cependant des problèmes. Wittgenstein a, ce me semble, adopté et adhéré à cette conception de la grammaire parce qu'elle paraissait garantir l'autonomie de l'usage du langage. Si l'usage du langage consiste à suivre des règles fixes et que ces règles sont arbitraires, alors l'usage du langage l'est également. Mais Wittgenstein a été forcé de prendre conscience de l'importance des formes de l'usage du langage qui sont dépourvues de règles, c'est-à-dire des formes qui ne consistent pas littéralement en la mise en œuvre de règles fixes. C'est le cas, apparemment, de certains gestes et de certaines formes primitives d'usage du langage qui font sens dans des circonstances appropriées ${ }^{25}$. D'un point de vue extérieur et dans un langage plus riche, le sens d'un geste peut être expliqué comme étant déterminé par des règles, mais si c'est là ce qu'on entend quand on dit qu'une forme primitive d'usage du langage consiste à suivre des règles fixes, alors cet usage du langage n'est pas autonome. Il ne saurait "parler pour lui-même » puisqu'il dépendrait de règles extérieures à lui-même. Il dépendrait d'une interprétation ou d'une théorie exprimée dans une forme d'usage du langage différente.

Mais l'idée de Wittgenstein au début des années trente est que la grammaire décrit l'usage effectif du langage, que la grammaire est manifeste et se présente elle-même de manière pleine et entière dans l'usage du langage, de sorte que l'autonomie de la grammaire du langage est l'autonomie de l'usage du langage.

La grammaire philosophique, en tant que morphologie descriptive et autonome de l'usage des expressions, est ce que Wittgenstein avait selon moi à l'esprit quand il annonça dans une conférence prononcée à l'automne I930 qu'il avait désormais une conception claire de ce qu'était la méthode correcte en philosophie. Comme le relate Ray Monk, Wittgenstein entama la conférence en question en affirmant sur un ton apocalyptique que «[1]'aura de la philosophie s'est perdue», et il ajouta que «nous sommes

25. Sur ce point, Wittgenstein semble avoir été fortement influencé par ses conversations avec P. Sraffa, voir McGuinness (2005), p. 337. 
actuellement dotés d'une méthode pour faire de la philosophie et pouvons parler de philosophes de métier ${ }^{26}$ ", signifiant par là qu'un changement notable ou même qu'un tournant s'était opéré dans sa pensée philosophique, lequel tournant marque le début de sa philosophie ultérieure.

Étant donné la manière dont Wittgenstein conçoit la grammaire à cette époque, l'explication d'une connexion entre le langage et une quelconque structure cachée d'une réalité externe, à savoir quelque chose qui serait à découvrir dans le futur (tel que, par exemple, les propositions élémentaires du Tractatus), ne pose aucun problème, et cela l'autorise à affirmer que: «[...] nous avons déjà tout, nous l'avons présentement, et il n'y a rien que nous devrions attendre. Nous nous mouvons dans le domaine de la grammaire de notre langue habituelle, et cette grammaire est déjà là ${ }^{27}$."

Wittgenstein considérait de ce point de vue l'attitude dogmatique du Tractatus comme sa faute principale, et le dépassement du dogmatisme est présenté ici comme l'aspect le plus important de ce qu'il appelle «la méthode correcte en philosophie ${ }^{28}$.

L'usage humain du langage, le langage parlé, est autonome en un sens important que Wittgenstein exprime au moyen d'une comparaison entre «cuisiner» et "parler ${ }^{29}$. Les règles de «cuisine» ne sont pas arbitraires au sens où le sont les règles de grammaire. La différence tient au fait que la finalité de la cuisine précède sa pratique et en constitue une précondition, tandis que le ou les buts de l'usage du langage sont internes à sa pratique, et n'existent pas indépendamment de celle-ci. On peut être tenté de dire que le

26. Monk, I990, p. 298. Les citations sont tirées des Cours de Cambridge 1930-1932, p. 24 .

27. Wittgenstein et le Cercle de Vienne, p. I63.

28. Je suis d'accord avec Marie McGinn quand elle décrit ce tournant dans la pensée de Wittgenstein comme un tournant «vers le phénomène concret du langage-en-usage » qui permet au langage lui-même de révéler son propre fonctionnement (McGinn 2006, p. 286). Il me semble cependant que ce tournant ne sera pas complété avant que Wittgenstein n'ait acquis une claire vision des problèmes liés au fait de suivre une règle, tels qu'ils seront abordés plus tard dans Recherches philosophiques. Un aspect problématique de la conception wittgensteinienne du calcul au début des années trente est l'idée que la compréhension d'une règle, ou le sens de l'expression d'une règle, précède l'usage du langage qui consiste à suivre cette règle. L'idée que cette compréhension soit une sorte de compréhension métalogique est une idée que Wittgenstein a eue auparavant, mais qu'il rejette au cours du tournant qui s'opère vers le début des années trente. Ce qui demeure cependant à cette époque est l'écart entre règle et application, la «faille infranchissable entre règle et application ou entre loi et cas particulier» comme Wittgenstein lui-même l'exprime dans les Remarques philosophiques, $\mathbb{1}$ I64, p. I89. C'est comme si cette faille pouvait seulement être colmatée par la découverte de quelque chose qui constitue la correction dans le fait de suivre la règle. Mais à mesure que cette idée de la constitution de la correction voit sa pertinence remise en cause à la lumière des considérations sur le fait de suivre une règle exposées dans les Recherches philosophiques, la conception du langage comme calcul perd une grande partie de son intérêt pour Wittgenstein au profit d'une conception du langage et des énigmes philosophiques moins formelle et plus «anthropologique».

29. Fiches, $\mathbb{3} 20$, p. 83 . 
but de l'usage du langage est d'exprimer et de communiquer des pensées, des désirs, des attentes, etc., mais l'usage effectif du langage est déjà présupposé par ces notions d'expression et de communication des pensées. C'est seulement sur l'arrière-plan constitué par le fait que nous avons le langage, que nous parlons, que nous possédons les notions en question.

On pourrait croire que le langage, à la différence d'un jeu inventé, est essentiellement connecté au monde réel d'une manière dont nombre de jeux ne sont pas, et que cette connexion repose sur le fait que plusieurs mots signifient ou réfèrent à des choses extérieures au langage. Mais qu'un mot ait une référence n'est pas un fait de nature. Le langage est un artefact humain, et ce qui confère ultimement à un mot son statut de mot n'est pas le fait qu'il a une référence ou une signification par lui-même en tant que simple son ou suite de lettres, mais le fait qu'il a un emploi dans le langage bumain. Un mot a une référence parce qu'il a un emploi dans le langage, et non le contraire. Par conséquent, l'autonomie de l'usage du langage n'est pas violée par le fait que le langage est rattaché au monde réel par le truchement de la référence des mots. On peut voir ainsi comment l'idée d'autonomie est en harmonie avec l'idée que «la signification d'un mot est son emploi dans le langage ${ }^{30} »$.

La signification d'un mot est son emploi dans le langage, et puisque cet emploi est autonome (c'est-à-dire qu'il n'est justifié par rien en dehors du langage), il est clair qu'il n'existe pas de chose telle que la signification qu'un mot doit avoir (par exemple, en tant que réflexion de la nature de la réalité, ou en tant qu'accord avec l'essence du concept signifié par le mot). Les mots ont seulement la signification qu'ils se trouvent avoir dans un certain emploi.

L'autonomie de la grammaire préserve ainsi du type de dogmatisme dont Wittgenstein lui-même s'est reconnu coupable dans le Tractatus, quand il se figurait un sens basique que certains mots devaient avoir afin de correspondre à la nature des choses, une sorte de signification fondamentale qui ne pouvait être différente de ce qu'elle était (par exemple, la signification de mots tels que "langage", "proposition", "objet", "nom»), et que cette signification était présupposée dans les grandes questions de la philosophie telles que: quelle est la nature d'une proposition?

C'est sans aucun doute cette tendance au dogmatisme que Wittgenstein avait à l'esprit quand il a affirmé (dans le Cahier bleu) que: «[p]our conjurer l'envoûtement de nos habitudes, nous tenterons également de construire de nouveaux systèmes de notation ${ }^{31}$ ».

Reconnaître l'importance de l'autonomie de la grammaire peut s'avérer difficile, entre autres pour la raison suivante: si cette idée est inspirée (comme je l'ai déjà suggéré et le montrerai plus en détail dans les sections suivantes) par certains courants des mathématiques modernes et des

30. Recherches philosophiques, $\mathbb{\$} 43$, p. 50 .

31. Le Cabier bleu, p. 78. 
sciences naturelles, pourquoi cela n'a-t-il pas rapproché Wittgenstein du scientisme ou du naturalisme (comme on aurait peut-être pu s'y attendre), mais au contraire a-t-il renforcé son opposition à l'emploi de méthodes scientifiques en philosophie? Car, encore une fois, les positivistes logiques ont également été inspirés par les mêmes courants des mathématiques modernes et des sciences naturelles.

L'inspiration que Wittgenstein a puisée dans les mathématiques et la science n'avait rien à voir avec les idées d' «explication » et de «reconstruction rationnelle ", c'est-à-dire avec l'emploi d'outils mathématiques pour la construction de nouveaux concepts en science destinés à remplacer des notions philosophiquement problématiques. Comme il a déjà été suggéré, la méthode grammaticale de Wittgenstein travaille dans la direction opposée, vers la source des notions problématiques; la clarification est visée pour ellemême et n'est pas au service de la science. Wittgenstein s'est intéressé tout particulièrement aux courants des mathématiques modernes et de la physique qui étaient motivés par un désir de dépasser le dogmatisme philosophique (sans pour cela conduire à un nouveau dogmatisme). Il a été inspiré et s'est senti stimulé par les efforts pour libérer la science des attitudes dogmatiques et des préjugés enracinés dans la philosophie métaphysique traditionnelle. De tels efforts sont visibles, par exemple, dans les travaux de Ernst Mach, Ludwig Boltzmann et Heinrich Hertz, ainsi que dans ceux des mathématiciens formalistes.

Les travaux de Mach, Boltzmann et Hertz avaient une visée scientifique, mais Wittgenstein en a reconnu la portée pour le langage en général: les attitudes et les préjugés qui plongeaient leurs racines dans le dogmatisme métaphysique avaient leurs contreparties et leurs équivalents dans notre conception du langage en général.

Dans le reste de cet article, nous examinerons quelques exemples plus spécifiques de l'affinité entre la méthode philosophique de Wittgenstein au début des années trente et certains courants des mathématiques modernes et des sciences naturelles.

\section{Grammaire et géométrie}

Déjà en I9I3, Wittgenstein notait dans un carnet la remarque suivante à propos de la grammaire et de sa signification pour la philosophie: «Se méfier de la grammaire est la première condition requise pour philosopher ${ }^{32}$.»

Lorsqu'il revient à la philosophie à la fin de l'année I920, la question de la nature de la grammaire se retrouve à plusieurs reprises dans ses écrits, et l'attention qu'il lui consacre est motivée par le désir d'éliminer les erreurs qu'il a repérées dans le Tractatus, en particulier l'idée que la nature logique de la proposition devrait pouvoir s'exprimer dans les termes du calcul des 
fonctions de vérité. L'idée qu'il souhaite explorer à la place est exposée dans les Remarques philosophiques comme suit:

Est complète l'analyse logique de la proposition dont la grammaire est complètement tirée au clair. Et cela quelle que soit la forme d'expression selon laquelle cette proposition se trouve écrite ou dite.

[...] Tout ce qui est possible et indispensable, c'est de séparer ce qu'il y a d'essentiel dans notre langage de ce qui y est inessentiel ${ }^{33}$.

Wittgenstein emploie ici l'expression "notre langage », non seulement par opposition à l'idée d'un langage logiquement idéal, mais également par opposition aux notions de «langage phénoménologique» ou de «langage primaire ", notions qu'il avait lui-même déjà utilisées auparavant avant de les rejeter.

À cette époque, il se situe encore pour l'essentiel à l'intérieur du cadre du Tractatus et se préoccupe de le réviser plutôt que de s'en dégager et de repartir sur d'autres bases. Il demeure sous l'influence de la distinction entre le langage comme représentation et la réalité comme ce qui est représenté, et de la conception selon laquelle l'essence des propositions est de représenter les états de choses de la réalité. Le langage et la réalité doivent par conséquent partager une forme logique. Ils doivent avoir la même "multiplicité » (une notion que Wittgenstein a empruntée à Hertz). Par exemple, une proposition montre que le fait qu'elle représente consiste en la combinaison de sept objets simples par la combinaison de sept noms distincts. Dans une conférence prononcée au cours du semestre du printemps 1930, Wittgenstein affirme la chose suivante:

La multiplicité du langage est donnée par la grammaire. Une proposition doit avoir la même multiplicité que le fait qu'elle exprime: elle doit avoir le même degré de liberté. Nous devons avoir la capacité de faire avec le langage autant que ce qui peut se produire en fait. La grammaire nous autorise à faire certaines choses avec le langage et non certaines autres: elle détermine le degré de liberté.

[...] [L]a grammaire n'est pas entièrement affaire de choix arbitraires. Elle doit nous permettre d'exprimer la multiplicité des faits, nous donner le même degré de liberté que le font les faits ${ }^{34}$.

Telle que la concevait Wittgenstein encore à cette époque, la grammaire n'est pas autonome.

Dans le courant de l'année I930-I93 I, Wittgenstein a expliqué à un étudiant le sens de quelques-unes des premières remarques du Tractatus et a fait la remarque suivante:

33. Remarques philosophiques, p. $5 \mathrm{I}$.

34. Les cours de Cambridge 1930-1932, p. 8-9. 
I.I3. «Les faits dans l'espace logique constituent le monde. » Espace logique a la même signification qu'espace grammatical. La géométrie est une sorte de grammaire: il y a une analogie entre grammaire et géométrie. L'espace grammatical inclut toutes les possibilités. "La logique traite de toutes les possibilités » $(2.0 \mathrm{I} 2 \mathrm{I})^{35}$.

Le lien entre la grammaire et la géométrie sur lequel Wittgenstein insiste ici est d'une grande importance dans le développement de sa conception de la grammaire au cours des années suivantes, et une bonne part de son travail à cette époque sera consacrée à l'examen de cette analogie. Ses réflexions sur la nature de la grammaire ont influencé sa conception de la nature de la géométrie, mais l'influence et l'inspiration sont encore plus fortes dans la direction opposée: la géométrie est fréquemment utilisée comme un modèle pour la grammaire. Dans les Remarques philosophiques, il dit: "Toujours géométrie et grammaire correspondent l'une à l'autre ${ }^{36}$." La remarque suivante indique qu'il était au fait des discussions animées sur la nature de la géométrie et sur son rôle en physique, lesquelles avaient cours dans les premières décennies du vingtième siècle, et que celles-ci l'ont influencé dans son travail:

La géométrie euclidienne appartient à la grammaire. Elle est une convention concernant l'expression, et par là, partie de la grammaire. (Minkowski rend compte des résultats de l'expérience de Michelson-Morley par une géométrie nouvelle, Fitzgerald par une contraction. Il s'agit là tout simplement de deux expressions du même fait; nous pouvons adopter indifféremment l'une ou l'autre; à moins qu'une expérience décisive ne fasse le partage entre elles ${ }^{37}$.)

Dans la Grammaire philosophique, nous retrouvons la remarque suivante sur la nature de la géométrie:

La géométrie n'est pas la connaissance (la science) des surfaces géométriques, des droites géométriques, des points géométriques, par opposition à une autre science qui traite en gros des droites, traits et surfaces physiques et énonce leurs propriétés. Ce qui fait la relation entre la géométrie et les propositions de la vie pratique relatives aux limites entre les couleurs, aux arêtes, aux angles, n'est pas qu'elle parle des mêmes choses, quoiqu'il s'agisse d'arêtes et d'angles idéaux; cela provient de la relation qui existe entre ces propositions et leur grammaire. La géométrie appliquée est la grammaire des énoncés concernant les objets spatiaux. Le rapport d'une prétendue droite géométrique à une limite de couleur n'est pas le rapport du raffiné au grossier, mais celui de la possibilité à la réalité ${ }^{38}$.

35. Les cours de Cambridge 1930-1932, p. I33.

36. Remarques philosophiques, $\mathbb{1}$ I $57, \mathrm{p}$. I77.

37. Les cours de Cambridge 1930-1932, p. 9.

38. Grammaire philosophique, deuxième partie, III, I7, p. 408-409. 
Ce que Wittgenstein remet en question ici est la conception commune et vulgaire de la géométrie (partagée même par certains mathématiciens éminents, comme par exemple Felix Klein) selon laquelle les figures et les diagrammes que nous traçons sur le papier ou le tableau noir ne sont que des approximations inexactes des objets géométriques exacts ou idéaux dont traite en réalité la géométrie, comme lorsqu'il est affirmé que «nous ne pourrons jamais tracer un cercle parfait ni en trouver un dans l'espace physique, de tels cercles seront toujours inexacts, tandis que les objets dont traitent les théorèmes de la géométrie sont exacts». Une telle conception a pour effet d'hypostasier les possibilités et d'en faire des sortes d'objets purement «idéaux». Mais pour Wittgenstein, le «cercle idéal » n'est pas littéralement un cercle, il est une règle de grammaire, une image grammaticale, qui détermine ce qu'il fait sens et ce qu'il ne fait pas sens de dire, à l'intérieur du cadre conceptuel de la géométrie euclidienne, à propos d'un cercle réel sur le papier ou dans l'espace physique ${ }^{39}$.

Le cercle que nous dessinons sur le papier dans l'énoncé et la preuve d'un théorème concernant les cercles en géométrie pure jouent le rôle d'une variable dans la preuve. Il s'agit, comme on dit, d'un "cercle arbitraire». C'est l'usage que nous faisons du cercle tracé dans la preuve qui en fait un "cercle arbitraire", et non pas le fait que le cercle tracé sur le papier réfère à un quelconque "objet idéal ", sur lequel le théorème "porterait ». Dans les applications du théorème à des cercles réels, un cercle réel est assigné comme valeur à la variable. Mais les cercles que nous traçons dans les preuves et les constructions en géométrie pure sont en même temps des cercles réels, auxquels s'appliquent des théorèmes déjà prouvés. Ce double rôle des figures et des diagrammes en géométrie, à la fois expressions d'une règle et instances de la règle, est l'une des bases de la «clarté » des preuves géométriques. La conception que se fait Wittgenstein de la clarté de la grammaire a été sans conteste influencée par la clarté des preuves géométriques, comme en témoigne son usage, fréquent à cette époque, de remarques grammaticales formelles telles que «toute barre a une longueur». Si cette remarque est prononcée en montrant une barre spécifique, alors cette barre joue un double rôle, celui d'une barre arbitraire et celui d'une instance de barre spécifique.

Souvenons-nous du diagramme familier figurant dans la preuve que la somme des angles d'un triangle est égale à deux angles droits. Il est clair que, dès que l'on comprend la preuve, on voit que le cœur de la preuve, sa clarté,

39. Bien entendu, Wittgenstein n'ignorait pas que l'image et que la description de la géométrie comme science des "objets idéaux" sont très courantes en mathématiques, où elles sont le plus souvent sans danger et peuvent même se révéler stimulantes pour l'invention. Mais il s'inquiète de la manière dont ce genre de description est une source potentielle d'erreur en philosophie des mathématiques, ce qui est souvent le cas lorsque les philosophes en viennent à parler de "possibilités en principe». 
est dans la construction du diagramme, et que les mots et les énoncés peuvent y être omis - y compris l'assertion qui en constitue la conclusion ${ }^{40}$. Le diagramme nous convainc que ce qui a été fait avec le triangle dans un tel diagramme peut être fait avec n'importe quel triangle. Ce n'est pas cette conviction en tant qu'état d'esprit qui importe le plus, ce sont les "applications qui justifient cette conviction ", comme le dit Wittgenstein ${ }^{41}$. C'est la conviction de pouvoir reproduire la construction du diagramme avec n'importe quel triangle. C'est en ce sens que la clarté d'une preuve géométrique influence la conception que se fait Wittgenstein de la clarté d'une investigation grammaticale.

Supposons que nous faisons de la géométrie euclidienne et que nous indiquons deux points distincts, $\mathrm{A}$ et $\mathrm{B}$, au tableau. Alors nous pouvons dire (avec justesse): "Il y a une seule ligne droite qui va du point $\mathrm{A}$ au point $\mathrm{B}$. » Quelqu'un de peu familier avec le discours géométrique pourrait dire (en un sens également avec justesse): "Je ne vois aucune ligne droite allant du point A au point B. Il n'y en a aucune avant qu'elle ait été tracée.» Le premier énoncé, en tant que règle de grammaire, veut dire par exemple qu'il y a du sens à parler de la ligne droite entre les points A et B, mais qu'il n'y a pas de sens à parler «des deux lignes droites distinctes entre les points $\mathrm{A}$ et $\mathrm{B}$ ».

La réification trompeuse des possibilités en géométrie (induite par la référence dans le discours à des "objets idéaux») trouve sa contrepartie dans la grammaire du langage en général, avec la conception selon laquelle les énoncés grammaticaux sont des sortes d'énoncés matériels concernant des états de choses dans un quelconque royaume conceptuel (caché), un royaume d'états de choses conceptuels qui constituerait pour la grammaire un fondement ou une réalité dont elle aurait la responsabilité de rendre compte. Mais la grammaire n'est responsable envers aucune réalité en ce sens.

Quand Wittgenstein affirme, dans le passage cité ci-dessus, que «la géométrie appliquée est la grammaire des énoncés concernant les objets spatiaux ${ }^{42}$ ", il est sûrement conscient du fait que la géométrie a été axiomatisée de manière à former un système axiomatique autonome dans lequel les concepts géométriques sont détachés, ou "coupés» de leur application. Là est peut-être la raison pour laquelle il parle de "géométrie appliquée » plutôt que simplement de "géométrie». Quoi qu'il en soit, ce résultat en géométrie, que l'on doit à Hilbert, a été sans nul doute l'un des facteurs qui ont permis à Wittgenstein de surmonter le préjugé qui lui faisait considérer que la grammaire était en quelque sorte responsable d'une réalité hors langage,

40. Comme le remarque Juliet Floyd: «"Voir” la clarté d'une preuve n'est pas la même chose que croire à la vérité de sa conclusion" (Floyd, 2000, p. 252) (NDLT: ma traduction).

41. Remarques sur les fondements des mathématiques, troisième partie, $\mathbb{2} 25$, p. I 5 I.

42. Grammaire philosophique, deuxième partie, III, I7, p. 409. 
plutôt que comme un calcul autonome ${ }^{43}$. Ce changement dans sa pensée a été radical, au point que sa conception de l'analyse logique s'en est trouvée modifiée en profondeur, comme en témoignent les remarques suivantes:

L'idée inexacte est que l'application d'un calcul dans la grammaire de la langue réelle le coordonne à une réalité, lui confère une réalité qu'il n'avait pas auparavant.

[...] Que se passe-t-il donc lorsqu'on trouve la relation à six places? Cela ressemble-t-il à la découverte d'un métal qui a les propriétés souhaitées (décrites auparavant, poids spécifique, solidité, etc.) ?

[...] Tout cela se trouve aussi en rapport avec le concept erroné de l'analyse logique que nous avions, Russell, Ramsey et moi-même. De telle sorte que l'on attend une analyse logique finie des faits, semblable à une analyse chimique des composants. Une analyse qui permet de trouver une relation à sept places tel un élément qui a effectivement le poids spécifique $7^{44}$.

Wittgenstein ne veut plus se laisser égarer par une analogie trompeuse. Par contraste, il déclare:

La grammaire est pour nous un pur calcul. (Ce n'est pas l'application d'un calcul à la réalité.)

[...] Car il ne s'agit pas ici d'une relation à la réalité qui maintiendrait la grammaire dans son ornière! La "relation du langage à la réalité » au moyen des définitions ostensives ne rend pas la grammaire contraignante (ne la justifie pas). Car celle-ci demeure toujours un simple calcul flottant librement dans l'espace, qui ne peut être que développé sans jamais recevoir aucun support physique $^{45}$.

S'étant rendu compte que l'idée d'une corrélation ou d'une harmonie entre le langage et la réalité, corrélation qui fixerait le degré de liberté de la grammaire, repose sur une fausse analogie, Wittgenstein ne peut plus se donner pour tâche de corriger ou de réviser le Tractatus. Trop de notions et d'idées dans le Tractatus, y compris l'esprit très dogmatique dans lequel il a été rédigé, sont rattachées à l'idée d'une forme commune entre le langage et la réalité. Wittgenstein a dû sentir que l'esprit dogmatique du Tractatus ne correspondait plus à l'esprit dans lequel il pouvait faire de la philosophie. Cet esprit dogmatique lui apparaît désormais comme incorrect et malhonnête, et je présume que c'était en partie ce qu'il entendait quand il déclarait que «la philosophie avait perdu son aura ${ }^{46} »$.

43. Hilbert, 1902 .

44. Grammaire philosophique, deuxième partie, III, I 5, p. 398-399. La question au sujet de la découverte de la relation à six ou sept places est liée au au problème énoncé dans le Tractatus, $5 \cdot 554-5 \cdot 554^{2}$.

45. Grammaire philosophique, deuxième partie, III, I 5, p. 399 et 4 I.

46. Wittgenstein n'a jamais aimé utiliser une terminologie philosophique technique, en particulier dans sa philosophie tardive. Les mots "grammaire» et "grammatical» constituent 


\section{La grammaire et la méthode axiomatique}

Malgré leurs vues divergentes sur la manière d'aborder les problèmes de fondement des mathématiques, il existe des ressemblances intéressantes entre la grammaire ou la méthode d'investigation grammaticale de Wittgenstein et la nouvelle méthode axiomatique de Hilbert, utilisée pour la première fois par ce dernier dans ses travaux sur les fondements de la

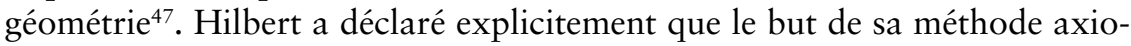
matique était d'exposer la structure logique des théories mathématiques et scientifiques existantes, et en particulier les théories des sciences naturelles existantes (au nombre desquelles il comptait la géométrie). La caractéristique de cette méthode que Hilbert appelle formelle, par opposition à la méthode génétique, est de permettre de concevoir les axiomes comme des stipulations formelles qui "définissent implicitement» la signification des mots et des signes utilisés. Cela n'est pas très éloigné de ce que dit Wittgenstein au début des années trente en ce qui concerne les règles de grammaire. Il affirme par exemple que la loi de la double négation $\neg\urcorner \mathrm{p}=\mathrm{p}$ «ne suit pas de la signification de "ne... pas", [elle] la constitue ${ }^{48}$ ". Il développe ce point dans la Grammaire philosophique, à nouveau par le biais d'une comparaison avec la géométrie: «La géométrie ne parle pas plus des cubes que la logique ne parle de la négation. Elle définit la forme cubique mais ne la décrit pas $^{49}$." Exprimer la forme logique de quelque chose n'est pas la décrire. La règle de la double négation n'est pas une assertion à propos de la négation, c'est une règle de remplacement d'un signe par un autre, ou une règle sur la manière dont nous effectuons des opérations avec le signe de la négation.

Mais on voit également en quoi diffèrent les méthodes de Hilbert et de Wittgenstein. Wittgenstein refusait, au contraire de Hilbert, de considérer les axiomes de la géométrie comme des propositions concernant un certain domaine de choses ou d'objets (non spécifiés). Wittgenstein était probablement d'avis que cette conception reposait en trop grande part sur une ana-

des exceptions. Ces termes sont utilisés fréquemment dans les Recherches philosophiques, souvent dans un esprit de satisfaction et de contentement, comme si Wittgenstein se rappelait quelque chose qui soit à la fois important et soulageant. Il est clair à la lecture de la plupart des passages où les mots "grammaire» et "grammatical» apparaissent qu'il fait allusion à des caractéristiques en rapport avec l'autonomie de la grammaire, plutôt qu'aux liens que ces termes entretiennent avec la grammaire traditionnelle en linguistique.

47. Voir Mülhölzer, 2005 et 2008 , pour d'autres similitudes intéressantes (et de différences) entre les conceptions respectives de Hilbert et de Wittgenstein sur les problèmes de fondement des mathématiques. Dans l'ensemble, on peut dire que Wittgenstein a été bien plus inspiré par les idées de Hilbert que ne l'ont en général reconnu les spécialistes de Wittgenstein. Cela ne signifie pas que Wittgenstein appuie le programme fondationnel de Hilbert. Mais je pense qu'on peut dire qu'il le trouve intéressant et utile, y compris pour les erreurs philosophiques qui s'y trouvent.

48. Les cours de Cambridge 1932-1935, p. I 5.

49. Grammaire philosophique, première partie, II, $\mathbb{I}$ I , p. 77 . 
logie problématique entre les propositions mathématiques et les propositions empiriques, et qu'en géométrie cette conception conduisait à considérer les propositions géométriques comme des énoncés descriptifs d' «objets idéaux». La géométrie euclidienne, en tant que système axiomatique, était plutôt pour Wittgenstein un pur calcul, c'est-à-dire un calcul permettant d'effectuer des opérations avec des expressions, y compris des figures et des diagrammes, et non pas de faire des assertions à propos de figures et de diagrammes!

Considérons par exemple le groupe d'axiomes dans l'axiomatisation de la géométrie euclidienne de Hilbert, que celui-ci appelle les axiomes


vons y lire l'axiome suivant:

Si $A, B$ et $C$ sont des points dans une ligne droite et que $B$ est entre $A$ et $C$, alors $B$ est aussi entre $C$ et $A$.

Si nous utilisons la notion wittgensteinienne de «règle de grammaire ", nous appellerons tout naturellement cet axiome une règle de grammaire pour le mot "entre» dans le langage de la géométrie euclidienne, ou une règle constitutive pour le concept euclidien d' "être entre". En tant que règle de grammaire, l'axiome énonce qu'il n'y pas de différence significative entre les expressions « le point $B$ est entre $A$ et $C$ » et «le point $B$ est entre $C$ et $A$ » dans le système de la géométrie euclidienne. Les deux expressions peuvent être substituées l'une à l'autre.

Cela nous fournit un exemple simple de ce que Wittgenstein voulait dire quand il parlait de rompre le charme des notions auxquelles nous sommes habitués. Cette règle pour la notion euclidienne d' «entre» n'était pas énoncée comme un axiome dans la formulation originale de la géométrie euclidienne, mais était probablement tenue pour acquise en raison de la «signification intuitive» du mot «entre» à laquelle on était accoutumé, même dans le discours non mathématique, et que l'on était par conséquent enclin à considérer comme une notion unique qui ne pouvait pas être différente. Comme si le mot "entre», non seulement se trouvait à signifier, mais devait signifier une relation symétrique; comme si la symétrie était implicite dans la signification du mot "entre", telle une "propriété intrinsèque». Nous avons ici un exemple simple du type de dogmatisme auquel j'ai fait allusion plus haut concernant la signification qu'un mot doit avoir, et avec lui la tentation de justifier ce "devoir» par une référence à une quelconque réalité, un royaume substantiel et non temporel comme la nature a priori de l'espace physique ou les formes a priori de l'intuition spatiale. En rendant la grammaire de la notion euclidienne $\mathrm{d}$ ' «entre » explicite en tant que règle de grammaire d'un système délimité, l'apparence d'unicité et d'immuabilité de

50. Hilbert I902, p. 5-6. 
la notion disparaît, tout comme la tentation de donner à sa signification une justification métaphysique.

Un autre exemple emprunté à Hilbert d'un axiome qui rend explicite quelque chose d'ordinairement tenu pour acquis dans la pratique scientifique est celui de l'axiome de continuité en physique. Cet axiome s'énonce comme suit:

Si pour la validité d'une proposition de la physique nous fixons un degré de précision quel qu'il soit, alors il est possible de spécifier des petites régions à l'intérieur desquelles les présupposés qui sous-tendent la proposition peuvent varier librement, sans que la déviation de la proposition dépasse le degré de précision prescrit ${ }^{51}$.

Hilbert affirme au sujet de cet axiome que

Cet axiome ne fait au fond rien de plus qu'exprimer quelque chose qui se trouve déjà dans l'essence de l'expérience; il est constamment présupposé par les physiciens, mais il n'avait pas été formulé auparavant ${ }^{52}$.

Cet axiome exprime également ce que Wittgenstein appellerait une règle de grammaire pour le langage de la physique expérimentale. Quand Hilbert lui confère le statut d'axiome exprimant l'essence de l'expérience, il précise que cet axiome a une fonction différente de la fonction des propositions empiriques de la physique; son rôle en tant que règle de grammaire est accentué.

Si nous laissons de côté la conception philosophique (néo-kantienne) de Hilbert concernant son axiomatique, qui est de «rendre explicite une strate plus profonde d'axiomes ", comme Hilbert le dit lui-même ${ }^{53}$, et que nous portons plutôt notre attention sur la manière dont il travaillait, il m'apparaît qu'une des caractéristiques essentielles de la méthode de Hilbert est qu'il s'autorisait à aborder les théories avec l'objectif de les représenter comme des systèmes autonomes: une théorie a été complètement axiomatisée seulement quand elle a été représentée comme un système autonome, comme un réseau de concepts détachés de leurs applications. Nous avons ici un exemple de ce dont parle Wittgenstein quand il dit: "[Considérer] les mathématiques comme nous le faisons [revient à] créer une section particulière - [à] séparer la mathématique pure de son application ${ }^{54}$.» Cette approche peut évidemment être comprise comme si les axiomes du système complètement axiomatisé avaient le statut de règles de grammaire arbitraires au sens où l'entend Wittgenstein. Les axiomes déterminent la manière dont nous effectuons des opérations avec des signes.

51. Hilbert, І9г 8, dans Ewald, I996, p. I I I0. (NDLT: ma traduction, d'après la version anglaise.)

52. Ibid.

53. Ibid., p. I I09...

54. Cours sur les fondements des mathématiques. Cambridge 1939, Cours XV, p. I 50. 


\section{Dans une lettre à Frege, Hilbert écrit:}

[I]l est bien évident que chaque théorie n'est qu'un échafaudage ou un schéma de concepts avec leurs relations réciproques nécessaires, et que les éléments de base peuvent être conçus de n'importe quelle manière ${ }^{55}$.

Si les «éléments de base", par exemple les mots "point», "ligne» et "plan » en géométrie, peuvent être interprétés «de n’importe quelle manière », c'est qu'ils sont traités comme de simples signes dans la théorie axiomatique en faisant abstraction de la signification spécifique que ces signes peuvent avoir dans une application du système en particulier. La seule «signification » qu'ont ces signes dans le système axiomatique est les propriétés et les relations formelles que leur confèrent les axiomes, lesquelles déterminent la manière dont nous effectuons des opérations avec les signes dans le système.

Mais Hilbert parle ici de «ce qu'est une théorie» d'un point de vue logique ou formel, en tant que système de "mathématique pure ", comme il le dit aussi parfois. Il ne parle pas de la géométrie en tant que discipline scientifique. Hilbert n'entend pas nier que la géométrie soit une science de l'espace physique. Il a été qualifié de formaliste au sens vulgaire du terme, selon lequel «les mathématiques ne sont que des jeux avec des signes sans signification ». Le fait que Hilbert ait été décrit erronément comme un formaliste en ce sens (par exemple par Brouwer, Bourbaki et d'autres) résulte probablement d'un malentendu concernant la nature et le but de sa méthode axiomatique: on l'a confondue avec une méthode ou une technique pour la formulation de nouveaux systèmes axiomatiques abstraits en tant que tels ${ }^{56}$,

55. F. Rivenc et P. de Rouilhan (dir.), Logique et fondements des mathématiques. Anthologie (1850-1914), p. 228.

56. À propos de ce courant algébrique abstrait, inspiré par l'axiomatique de Hilbert, Corry, 2000, p. 52, écrit: "[D]ans les années qui ont immédiatement suivi la publication des Grundlagen, de nombreux mathématiciens, en particulier aux États-Unis, ont entrepris une analyse des systèmes des postulats abstraits pour des concepts algébriques tels que les groupes, les champs, l'algèbre booléenne, etc., basée sur l'application de techniques et de conceptions semblables à celles développées par Hilbert dans son étude sur les fondements de la géométrie. Rien ne prouve que Hilbert se soit intéressé à ce type de travaux, et il y a en fait des raisons de croire que ceux-ci impliquaient une direction de recherche que Hilbert n'a pas envisagée en proposant son programme axiomatique. Il semble raisonnable d'affirmer que Hilbert a même considéré que cette direction de recherche était mathématiquement mal conçue.» J'ai tendance à être d'accord avec Corry ici, et la conception selon laquelle Hilbert serait un formaliste me semble reposer dans une large mesure sur cette autre conception selon laquelle ce courant algébrique abstrait se trouvait en harmonie avec les objectifs du programme axiomatique de Hilbert. Mais je suis convaincu que ce n'était pas le cas. Les tenants de ce courant ne partageaient pas les objectifs épistémologiques et la visée de clarification de l'axiomatique de Hilbert. La conception des métamathématiques d'Alfred Tarski en tant que "méthodologie des sciences déductives» est également un exemple d'entreprise à situer dans le même esprit que ce courant algébrique abstrait, mais qui est incompatible avec les objectifs épistémologiques du programme de Hilbert. L'ouvrage de Van der Waerden, Moderne Algebra, en est un autre exemple. Ce dernier semble avoir été une importante source d'inspiration pour le programme de Bourbaki. 
alors qu'elle est une approche méthodologique pour l'exposition de la structure logique des théories scientifiques existantes, dans lesquelles les signes ont déjà une signification (qui demande toutefois à être clarifiée).

Il existe un malentendu analogue concernant la méthode grammaticale de Wittgenstein, selon lequel celle-ci serait une "philosophie linguistique " ou une "philosophie du langage ordinaire» (dans l'esprit de l'école d'Oxford), plutôt qu'une méthode pour l'investigation conceptuelle de concepts que nous possédons et que nous utilisons déjà, mais qui exigent d'être clarifiés, et où cette exigence est motivée par une confusion ou une énigme conceptuelle spécifique.

Hilbert n'était pas un formaliste en ce qui a trait à la géométrie en tant que discipline scientifique, pas plus que ne l'était Wittgenstein ${ }^{57}$. Lors d'une discussion avec Waismann, Wittgenstein a fait la remarque suivante: "La géométrie n'est pas quelque chose qui tient tout seul, elle est complétée par la physique. Elle est donc une partie d'une hypothèse ${ }^{58}$.» Leo Corry rapporte que Hilbert a avoué (dans une conférence de 1905) qu'il tendait à accorder à la géométrie euclidienne un statut préférentiel entre toutes les géométries mathématiquement possibles, mais qu'il lui semblait difficile d'en donner la raison ${ }^{59}$. Wittgenstein a fait une remarque similaire. Dans le cadre d'une discussion sur le concept de "simulation", et sur la manière dont il se manifeste dans le comportement humain, il fait la remarque suivante:

N'y a-t-il pas ici quelque chose d'analogue au rapport entre la géométrie euclidienne et l'expérience sensorielle? (Je veux dire: l'existence d'une profonde similitude.) Car la correspondance entre la géométrie euclidienne et l'expérience n'est pas du tout facile à comprendre et ne se comprend pas seulement sur un mode qui irait du plus exact au moins exact ${ }^{60}$.

Il n'est pas évident de savoir quelle ressemblance entre les manifestations de simulation et la géométrie euclidienne Wittgenstein avait ici à l'esprit. Peut-être voyait-il une ressemblance entre les deux façons de voir (problématiques) suivantes: "Toutes les géométries ont un statut égal» et "Tout comportement humain peut toujours être une simulation"?

\section{La grammaire et la théorie de la relativité}

Même si ni Hilbert ni Wittgenstein n'étaient des formalistes, il me paraît clair qu'ils ont tous deux trouvé la perspective formaliste en mathématiques très utile d'un point de vue méthodologique pour l'élaboration de leur méthode respective de clarification conceptuelle. La caractéristique la plus

57. Si Hilbert était un formaliste, alors le sens le plus correct du mot «formalisme» serait selon moi celui formulé par Detlefsen, I993a et I993b.

58. Wittgenstein et le Cercle de Vienne, p. I40-I4I.

59. Corry $2006 \mathrm{a}$, p. 165.

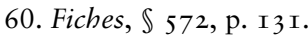


intéressante de la méthode axiomatique de Hilbert, si on la compare à la méthode d'investigation grammaticale de Wittgenstein, a été décrite par Einstein comme suit:

Le progrès réalisé par l'axiomatique consiste à avoir séparé de façon nette le logico-formel de son contenu objectif ou intuitiff ${ }^{61}$.

Comparons cette remarque à la remarque suivante de Wittgenstein: "[Considérer] les mathématiques comme nous le faisons [revient à] créer une section particulière - [à] séparer la mathématique pure de son application $^{62}$.» Ce que Wittgenstein décrit par le mot «séparer» est ce qu'Einstein décrit par le mot «séparation".

La méthode axiomatique de Hilbert (appelée également «axiomatique ») et la nouvelle axiomatisation de la géométrie semblent avoir joué un rôle décisif dans la formulation de la théorie de la relativité. Après avoir expliqué ce que le passage cité ci-dessus signifie pour la géométrie, Einstein va jusqu'à déclarer: "J'accorde une importance particulière à la conception de la géométrie que je viens de présenter, parce que sans elle j'aurais été incapable de formuler la théorie de la relativité63.»

Grâce à la séparation (comme l'appelle Einstein), la notion traditionnelle d'axiome en mathématiques (en tant que proposition ou jugement exprimant une vérité nécessaire et universelle sur un sujet quelconque) a été transformée en une règle que nous suivons dans un système, une règle qui a une nécessité interne seulement dans ce système. Les signes n'ont pas de sens ou de signification obligatoire, excepté à l'intérieur d'un certain système. Nous sommes libres de changer le sens des signes en changeant les axiomes, et d'obtenir ainsi un système mathématique différent. Les géométries non euclidiennes étaient des constructions de nouveaux systèmes mathématiques de ce type.

Einstein insiste entre autres (et davantage que Hilbert) sur un aspect particulier de la méthode axiomatique, à savoir la liberté que nous avons de changer la signification des signes en changeant leurs règles d'utilisation. Il s'est ainsi soustrait à la conception selon laquelle des mots tels que "espace ", «temps », et "simultanéité » ont une signification traditionnelle, absolue ou unique qu'ils doivent avoir dans tout usage. Einstein a même parlé du "caractère fictif des principes fondamentaux ", signifiant par là que le statut de "principes fondamentaux» s'est transformé, passant de «lois éternelles de la nature » à "règles arbitraires de la grammaire » (même si Einstein ne s'est pas exprimé en ces termes). L'essentiel est désormais la manière dont nous utilisons les notions, par exemple la manière dont nous déterminons effectivement la vérité d'un énoncé concernant la simultanéité de deux évé-

61. Einstein, $\mathrm{I} 973$, p. 233 (NDLT: ma traduction, d'après la version anglaise.)

62. Cours sur les fondements des mathématiques. Cambridge 1939, Cours XV, p. I 50.

63. Einstein, I973, p. 235 (NDLT: ma traduction, d'après la version anglaise.) 
nements. Einstein décrit cette liberté des systèmes conceptuels inventés comme la différence décisive entre l'ancienne philosophie de la nature et la manière de penser qui donne naissance à la théorie de la relativité:

Les philosophes naturels des époques précédentes, au contraire, étaient pour la plupart imprégnés de l'idée que les concepts fondamentaux et les postulats de la physique n'étaient pas au sens logique des inventions libres de l'esprit humain, mais pouvaient être déduits de l'expérience par "abstraction»c'est-à-dire par des moyens logiques. Le caractère erroné de cette conception est clairement apparu seulement avec la théorie de la relativité ${ }^{64}$.

Wittgenstein a sûrement eu connaissance des travaux d'Einstein, même si ce n'était pas de manière très détaillée. Les conceptions d'Einstein en géométrie ont fait l'objet de discussions aux réunions du Cercle de Vienne à l'époque où Wittgenstein y participait. Il a été sans nul doute impressionné par certains aspects des travaux d'Einstein, en particulier ceux que j'ai mentionnés. Dans la Grammaire philosophique, il écrit avec approbation: «Einstein: ce qu'est une grandeur, c'est la façon dont elle est mesurée ${ }^{65}$ ». Et déjà en I929-I930, alors qu'il se penche sur la relation entre l'attente et la satisfaction, Wittgenstein se réfère de manière explicite à la théorie de la relativité:

Mon idée est toujours que si quelqu'un pouvait voir l'attente, il verrait nécessairement ce qui est attendu.

L'attente est comme si elle était elle-même langage et ne pouvait sortir d'ellemême. (La ressemblance entre ma conception et celle de la théorie de la relativité réside dans le «ne pas pouvoir sortir de soi-même ${ }^{66}{ }^{*}$.)

Le "ne pas pouvoir sortir de soi-même » est un des traits principaux de l'autonomie d'un calcul, d'un jeu de langage ou de ce que Wittgenstein appelle aussi un système. Wittgenstein mentionne également la théorie de la relativité dans le cadre d'une discussion sur le signe d'assertion chez Frege. Il conclut son argument en disant: "La façon de penser [Denkbewegung] qu'il faut à nouveau ici est la façon de penser de la théorie de la relativité67.

En faisant ressortir certaines ressemblances entre la méthode axiomatique de Hilbert, la théorie de la relativité d'Einstein et la conception originale de la grammaire de Wittgenstein, je n'entends pas suggérer que leurs méthodes respectives avaient la même motivation et le même objectif. On peut certes affirmer qu'ils recherchaient tous la clarté conceptuelle, mais la

64. Einstein, I973, p. 273. (NDLT: ma traduction, d'après la version anglaise.)

65. Grammaire philosophique, deuxième partie, VII, 39, p. 585.

66. MS ı08, p. 27I. Cité d'après Hilmy I987, p. I46. La phrase sur la théorie de la relativité est reprise dans le Grand Cahier (The Big Typescript, TS 213, p. 355-356). (NDLT: ma traduction, d'après la version anglaise.)

67. MS I09, p. I99. (NDLT: ma traduction, d'après la version anglaise, et une remarque similaire faite par Wittgenstein dans De la certitude, $\mathbb{S} 305$, p. 83.) 
notion hilbertienne de clarté, à la différence de la notion wittgensteinienne, était fortement influencée par l'élan vers le progrès de la science moderne. La clarté pour Hilbert, aussi bien que pour Einstein, constituait sans nul doute un moyen pour cette fin, alors que pour Wittgenstein la clarté était une fin en soi. Pour Hilbert, de même que pour Frege et Russell, la logique était une discipline scientifique, et Hilbert employait sa méthode axiomatique, non dans un but de clarification philosophique au sens où l'entendait Wittgenstein, mais plutôt afin de contribuer au développement du courant algébrique moderne en mathématiques, dans la foulée de Dirichlet, Riemann, Dedekind, Weierstrass, Cantor et d'autres.

Si leurs travaux n'avaient ni le même motif ni le même objectif, les ressemblances que j'ai suggérées ne sont-elles pas complètement fantaisistes et superficielles? - Ce que je veux suggérer ici est que Hilbert avait le sentiment (tout comme Mach, Boltzmann, Hertz et Einstein) qu'il était nécessaire, pour comprendre, apprécier et agréer cette nouvelle orientation en mathématiques et en physique, de dépasser les façons de voir dogmatiques dominantes concernant les notions et les méthodes traditionnelles auxquelles étaient habitués de nombreux mathématiciens et scientifiques. C'est dans l'effort mis à surmonter les vestiges de ces façons de voir traditionnelles, dans un esprit en quelque sorte moderniste, que nous pouvons trouver une affinité évidente entre le travail de Hilbert et celui de Wittgenstein. Nous retrouvons par exemple cet esprit dans leur rejet commun de l'apriorisme kantien. Wittgenstein affirme déjà dans le Tractatus que: "Tout ce que, d'une manière générale, nous pouvons décrire, pourrait aussi être autre. Il n'y a aucun ordre a priori des choses ${ }^{68}$. $»$ Et à propos de la notion newtonienne de temps absolu, Hilbert déclare sur un ton polémique:

Kant, le philosophe critique, n'est pas du tout critique ici, car il a simplement accepté Newton. Seul Einstein nous a définitivement libéré de ce préjugé [...] $[\mathrm{L}] \mathrm{a}$ théorie kantienne de l'apriori charrie encore des scories anthropologiques dont il faut la libérer $[\ldots]^{69}$.

68. Tractatus, 5.634, p. 94 .

69. Mais Hilbert ne rejetait pas l'apriorisme kantien dans son entier. Je pense qu'il était très sérieux quand il poursuivait en ajoutant dans la même phrase: «Au final, seule l'attitude $a$ priori demeure, qui sous-tend également la connaissance mathématique pure: c'est essentiellement l'attitude finitiste que j'ai décrite dans plusieurs ouvrages " (Hilbert I930, citation tirée de Ewald I996, p. I I63) (NDLT: ma traduction, d'après la version anglaise). Les commentateurs du programme fondationnel de Hilbert ont tendance à ignorer cette remarque et d'autres similaires. Je ne pense pas que Hilbert ait insisté sur ce point aussi souvent uniquement dans le but de "rendre un hommage mérité à une figure dominante, celle de son concitoyen de Königberg " (Corry, 2006a, p. I 59) (NDLT: ma traduction, d'après la version anglaise). 


\section{Le solipsisme et la méthode des éléments idéaux}

Je considérerai encore un autre exemple qui montre, ce me semble, que Wittgenstein a puisé une inspiration dans les mathématiques modernes pour ses recherches grammaticales en dehors du champ des mathématiques. Mais cela ne veut pas dire qu'il faisait ou proposait un certain type de mathématiques appliquées comme en physique ou en psychologie mathématique; il utilise plutôt certaines analogies afin de "rompre le charme" des notions auxquelles nous sommes habitués.

Dans un passage du Cahier bleu ${ }^{70}$, Wittgenstein nous rappelle qu'un verbe psychologique comme, par exemple, "éprouver de la crainte», est utilisé tant d'une manière transitive que d'une manière intransitive. Nous pouvons craindre quelque chose ou quelqu'un, mais il arrive aussi que nous ayons simplement un sentiment de crainte sans pouvoir dire ce que nous craignons. Wittgenstein suggère comme expérience de pensée que nous pourrions introduire une terminologie nouvelle, plus simple et plus unifiée, dans laquelle les verbes psychologiques seraient toujours utilisés de manière transitive. Selon cette proposition, quand quelqu'un éprouve simplement de la crainte, sans savoir ce qu'il craint, il y a toujours quelque chose qu'il craint même s'il ne peut dire ce que c'est. Dans le même esprit, il suggère qu'on pourrait imaginer des situations où il serait pratique d'utiliser une terminologie dans laquelle nous pourrions parler de douleurs inconscientes, comme un «mal de dents inconscient». Wittgenstein affirme qu'il s'agit là du sens dans lequel la psychanalyse parle de pensées inconscientes, de volitions inconscientes, etc., et qu'il n'y a rien de fautif dans cette convention terminologique, tant que nous sommes capables de nous en servir sans la confondre avec la terminologie à laquelle nous sommes habitués. Une confusion de ce type se révélerait dans des énigmes philosophiques telles que: " $\mathrm{La}$ douleur inconsciente existe-t-elle vraiment? ? et "Comment un mal de dents inconscient est-il effectivement possible?».

Il existe une analogie frappante entre la construction de nouvelles terminologies de ce type chez Wittgenstein et la méthode de construction de nouveaux systèmes de notation mathématique utilisée et promue par Hilbert. Ce dernier appelait cette méthode la méthode des éléments idéaux. Un des exemples les plus connus de cette méthode est le système des nombres complexes, dans lequel les nombres complexes imaginaires constituent les éléments idéaux tandis que les nombres réels complexes constituent les éléments réels. Un trait de ce système de notation, en même temps qu'un avantage, est qu'il simplifie les théorèmes concernant l'existence et le nombre de racines d'une équation. Un autre exemple est celui d'un système de notation de la géométrie dans lequel des lignes de longueur infinie et des points à

70. Le Cahier bleu, p. 76 et suivantes. Voir aussi les Remarques philosophiques, $\mathbb{S} 58$. 
l'infini sont introduits comme éléments idéaux. Cette dernière construction a pour intérêt de simplifier le traitement algébrique de la géométrie ${ }^{71}$.

Dans la comparaison que j'établis ici (entre les nouveaux systèmes de notation mathématique et les systèmes de notation psychologique tels que proposés par Wittgenstein), les "douleurs inconscientes ", les "pensées inconscientes» et les «sentiments inconscients» constitueraient les «éléments idéaux", et les énigmes philosophiques causées par la confusion entre la nouvelle terminologie psychologique et la terminologie ordinaire pourraient être mises en parallèle avec des énigmes analogues concernant l'existence et la possibilité des nombres imaginaires et des points à l'infini en philosophie des mathématiques, comme Hilbert en était bien conscient. C'est la raison pour laquelle ce dernier a parlé d'éléments idéaux, par opposition aux entités réelles. L'utilisation de la méthode des éléments idéaux était effectivement une idée centrale dans le programme fondationnel de Hilbert. Toutefois, il ne s'agissait pas chez lui d'une simple analogie; cette idée lui permettait de définir sa conception des métamathématiques comme forme de mathématiques.

Wittgenstein utilise quant à lui cette analogie quand il traite du problème du solipsisme dans le Cabier bleu. Plutôt que de rejeter simplement, de manière dogmatique, la déclaration du solipsiste affirmant: "Je suis le seul à souffrir réellement " comme insensée et absurde, il suggère que nous pouvons faire sens de cette expression en la prenant comme proposition pour une nouvelle grammaire, c'est-à-dire comme règle grammaticale pour une nouvelle terminologie concernant l'usage des termes mentaux, une terminologie dans laquelle le solipsiste se voit octroyer une position centrale. La confusion du solipsiste apparaît comme telle quand il pense qu'il peut justifier sa nouvelle terminologie en arguant qu'elle est "plus correcte » que notre usage ordinaire des termes mentaux, tout comme si la nouvelle grammaire était plus "fidèle à la réalité". Le solipsiste confond alors les deux terminologies, et prend un énoncé grammatical pour une sorte d'énoncé matériel.

Il n'est pas invraisemblable que Wittgenstein se soit inspiré de ce que Hilbert a appelé la méthode des éléments idéaux pour proposer ses propres systèmes de notation psychologique, mais bien entendu cette méthode n'était pas une invention de Hilbert. Wittgenstein a pu en avoir connaissance par quelqu'un d'autre ${ }^{72}$. Je m'intéresse de toute manière en premier lieu ici aux idées des mathématiques modernes susceptibles d'avoir inspiré Wittgenstein.

71. Hilbert, I926, p. I95.

72. Jahnke (I993, p. 279) signale que même le poète romantique Novalis fait allusion à la méthode des éléments idéaux dans une tentative de caractériser le génie mathématique. 


\section{Wittgenstein et Hertz}

Bien qu'elles soient toutes deux des méthodes de clarification conceptuelle, il existe des différences importantes entre la méthode axiomatique de Hilbert et la grammaire de Wittgenstein. Cela tient au fait que Wittgenstein n'était ni un mathématicien ni un scientifique, et qu'en tant que philosophe il ne partageait pas la perspective néo-kantienne de Hilbert ${ }^{73}$. Mais il semble y avoir suffisamment de similitudes pour soulever la question de savoir s'il avait lu Hilbert. Il semblerait que Wittgenstein ait lu plusieurs des textes de Hilbert sur les fondements des mathématiques, ceux-là mêmes qui ont servi de base aux discussions du Cercle de Vienne auxquelles le premier a participé. La méthode axiomatique de Hilbert était en vogue à l'époque, notamment grâce aux travaux d'Einstein. Par conséquent, Wittgenstein la connaissait certainement, aussi grâce aux conversations qu'il avait eues avec Waismann, Schlick, Ramsey et d'autres. Mais l'explication de ces similitudes entre Wittgenstein et Hilbert qui me semble la plus probable, c'est qu'ils ont puisé leur inspiration aux mêmes sources pour leurs travaux de clarification conceptuelle, à savoir chez Ludwig Boltzmann et en particulier chez Heinrich Hertz. Comme l'a bien montré Leo Corry ${ }^{74}$, Hilbert a été influencé à plusieurs égards, en ce qui concerne le développement et l'usage de sa méthode axiomatique dans ses travaux en mathématiques et en physique théorique, par l'ouvrage de Heinrich Hertz, The Principle of Mechanics. L'influence de Hertz sur Wittgenstein est plus connue, étant donné que les références au livre de Hertz sont parmi les seules à figurer dans le Tractatus. Mais nombre de spécialistes de Wittgenstein ont tendance à sousestimer l'étendue de cette influence. Je suis enclin à abonder dans le sens de Gordon Baker et d'autres qui ont soutenu que l'influence de Hertz est également présente dans la philosophie tardive de Wittgenstein ${ }^{75}$.

73. La préface des Remarques philosophiques montre en outre clairement que Wittgenstein n'avait pas une foi aussi grande que celle de Hilbert en la science moderne.

74. Corry, 2006b.

75. Baker, I988. Voir aussi Barker, I980, Wilson, I989, Visser, I999 et Kjaergaard, 2002.

En novembre 1946, Wittgenstein a prononcé une conférence intitulée Philosophy au Cambridge Moral Science. Cette conférence était une réponse à une conférence donnée trois semaines auparavant par Karl Popper, dans laquelle ce dernier critiquait Wittgenstein en tant que figure de proue de «l'école de philosophie linguistique de Cambridge». Dans une lettre à G.E. Moore, Wittgenstein a décrit le contenu de sa conférence comme suit: "Je donne une conférence, en gros, sur ce qu'est la philosophie à mon sens, ou ce qu'est la méthode en philosophie" (PPO, p. 338) (NDLT: ma traduction). Selon les minutes de la rencontre, Wittgenstein s'est montré mécontent du fait que sa philosophie ait été décrite comme une "philosophie linguistique». Il a cité Hertz avec grande approbation, en le présentant comme sa principale source d'inspiration pour sa propre conception des problèmes philosophiques et sa manière de les traiter. Il a aussi mentionné Mach. Mais le nom d'aucun autre philosophe, pas même celui de Frege, ne semble avoir été prononcé dans cette conférence (PPO, p. 342). 
Le fait que Wittgenstein avait l'intention de mettre une citation tirée du livre de Hertz en exergue de la première version des Recherches philosophiques conforte ce point de vue. La citation en question était la suivante:

Si on lève ces contradictions douloureuses, la question de l'essence [die Frage nach dem Wesen - c.-à-d. dans le texte de Hertz, non repris dans l'épigraphe: de la force] n'est pas pour autant résolue; mais l'esprit cesse de se tourmenter et de se poser cette question illégitime ${ }^{76}$.

L'expression "contradictions douloureuses » fait référence aux controverses concernant la nature du concept de force en mécanique newtonienne, laquelle est une notion de base en mécanique classique. Comme le fait observer Peter Barker, l'erreur fondamentale des défenseurs de Newton, selon Hertz, était de penser que chaque terme d'une théorie scientifique correspond à une entité dans le monde ${ }^{77}$. Hertz soutenait que la question de la nature de la force est confuse parce qu'elle prend source dans la conception suivant laquelle le concept de force est forcément issu d'un contenu de base, un contenu justifié par la réalité physique et qui demeure à découvrir. Selon Hertz, la confusion « réside dans les caractéristiques inessentielles que nous avons introduites nous-mêmes de façon arbitraire dans le contenu essentiel donné par la nature ". Les problèmes ne sont pas liés au contenu de la notion en question, "mais seulement à la forme sous laquelle le contenu est représenté $^{78}$ ». Hertz a montré comment reformuler la mécanique classique sans utiliser la force comme notion de base. Aucune entité dans le monde ne correspond au mot «force». Hertz appelle cela une «relation vide» ou une "cinquième roue». Wittgenstein a utilisé la même métaphore dans ses écrits, y compris ses écrits tardifs.

Il n'a pas seulement retenu de Hertz l'idée générale de la résolution par la dissolution des énigmes philosophiques; il a été également influencé par la manière dont Hertz accomplissait cette tâche en reformulant et en recomposant les notions et les expressions de la mécanique classique. Hertz a décrit cet aspect de son travail comme suit:

[L]es défauts existants ne sont que des vices de forme; [...] le manque de distinction et de certitude peut être évité par une formulation appropriée des définitions et des notations et un mode d'expression soigné ${ }^{79}$.

Dans le Grand Cahier, Wittgenstein écrit :

Dans ma manière de faire de la philosophie, le but ultime est de donner à une expression une forme telle que certaines inquiétudes disparaissent (Hertz) ${ }^{80}$.

76. Hertz I 894, tel que cité par É. Rigal dans l'apparat critique des Recherches philosophiques, p. 327.

77. Barker I988, p. 245.

78. Hertz 1956 , p. 8. (NDLT: ma traduction, d'après la version anglaise.)

79. Ibid., p. 9. (idem).

80. MS 2I3, p. 42I. (NDLT: ma traduction, d'après la version anglaise.) 
L'idée de l'autonomie de la structure logique a rendu possible cette manière de travailler en nous donnant le contrôle sur nos représentations et la liberté de changer et de réordonner le système conceptuel. Cette idée est visiblement inspirée de la conception hertzienne de l'autonomie du système conceptuel de la mécanique et de la conception générale de Hertz de la théorie scientifique comme image et comme système symbolique. Un tel système constitue une entité autonome du point de vue formel et demeure indépendant des phénomènes empiriques qu'il sert à expliquer. Par « les principes de la mécanique ", précise Hertz, nous entendons: «toute suite de propositions semblables, telle qu'elle satisfasse la condition selon laquelle la totalité de la mécanique peut être développée à partir de cette suite par un raisonnement purement déductif sans recours additionnel à l'expérience ${ }^{81} »$. Cette conception est manifestement proche de l'idée hilbertienne d'une théorie comme réseau de concepts reliés par des relations purement logiques. Et la caractéristique de l'axiomatique de Hilbert sur laquelle insiste Einstein, à savoir celle d'avoir "séparé le logico-formel de son contenu objectif ou intuitif », est déjà présente chez Hertz.

Sous l'influence de Hertz, l'idée d'autonomie est déjà présente dans la notion tractarienne suivant laquelle la logique des propositions ne représente pas, la syntaxe logique ne doit pas référer au sens (Bedeutung) des expressions, et, plus généralement, dans l'idée suivant laquelle la "logique doit prendre soin d'elle-même ${ }^{82} »$.

On pourrait cependant soutenir que Wittgenstein, dans sa philosophie première, a été sur un certain point trop influencé, ou même induit en erreur, par les conceptions de Hertz. Une théorie scientifique est pour Hertz une image, un système symbolique, lequel est une entité autonome seulement dans son aspect formel. Un tel système est indépendant des phénomènes empiriques qu'il sert à expliquer seulement du point de vue formel ou logique. Mais la mécanique classique est une théorie qui porte sur des phénomènes empiriques donnés de manière indépendante. Il s'agit d'une construction destinée à servir un but, à savoir celui de représenter et d'expliquer des observations expérimentales, et, comme la cuisine (qui est aussi destinée à servir un but), elle n'est pas autonome sous ce rapport. Les conditions d'un système conceptuel de la mécanique que Hertz appelle sa correc-

81. Hertz, I956, p. 4, l'italique est de Stenlund. (NDLT: ma traduction, d'après la version anglaise.)

82. Je suis d'accord avec Marie McGinn quand elle écrit: «L'un des thèmes centraux des Recherches philosophiques réside dans la tentative de montrer que, dans la mesure où une phrase est utilisée pour exprimer une proposition grammaticale, elle ne représente pas (elle ne peut pas être comparée avec la réalité pour établir sa vérité ou sa fausseté): il n'y a rien qui fonde ou qui justifie la grammaire. [...] [L]e souci d'exposer l'autonomie de la grammaire rappelle le souci plus ancien de Wittgenstein de montrer que la logique ne représente pas - que "la logique prend soin d'elle-même" - et cela révèle une continuité profonde sur le plan du but philosophique» (McGinn, 2006, p. 295). (NDLT: ma traduction.) 
tion et sa convenance concernent les manières dont le système sert son but. Dans sa philosophie première, Wittgenstein semble avoir été amené à penser qu'il devait y avoir quelque chose de semblable à ces conditions également pour le langage humain en général. Dans le Tractatus, les conditions en question prennent la forme de la structure logique et de la multiplicité commune au langage et à la réalitée ${ }^{83}$. Wittgenstein est demeuré sur ce point sous l'influence de Hertz aussi tardivement que jusqu'au semestre du printemps I930. Il inclinait à voir le langage humain comme un système de représentation et affirmait que «la grammaire n'est pas entièrement affaire de choix arbitraires $[\ldots]$ ». Mais moins d'un an après, l'idée de l'autonomie de la grammaire du langage ordinaire une fois clarifiée, il rejetait cette première conception comme trompeuse.

L'ouvrage de Hertz peut paraître un ouvrage scientifique plutôt que philosophique, un traité de mécanique, mais il est clair que Hertz lui-même considérait que sa visée principale était philosophique. Dans la préface, il écrit: «Ce qui je l'espère est nouveau, et ce à quoi seulement j'attache de la valeur, c'est la construction et l'organisation de l'ensemble - l'aspect logique ou philosophique de la question.»

Pour Wittgenstein, il semblait évident que la suggestion de Hertz sur la manière d'éliminer les "contradictions douloureuses" qui nous poussent à poser des questions sous la forme "Quelle est la nature de X?" avait une application beaucoup plus grande et ne devait pas se limiter aux seules notions problématiques de la mécanique. Mais ce ne sont pas toutes les questions de cette forme qui constituent des énigmes ou des mystères. Hertz demande de façon rhétorique: "Mais pourquoi ne demande-t-on jamais en ces termes quelle est la nature de l'or, ou quelle est la nature de la vélocitéé ${ }^{84}$ »

Dans le cadre de l'attitude dogmatique qui était celle de la philosophie traditionnelle, éclaircir les mystères philosophiques signifiait se persuader soi-même d'avoir trouvé la réponse finale et définitive à la question de la nature ou de l'essence de X. Mais nombre de réponses proposées pour ces questions par le passé peuvent être considérées comme issues de formes spécifiques de dogmatisme, et l'Histoire montre qu'en général de telles réponses ne sont pas finales et définitives. L'idée de Hertz était que la réponse dont nous avons besoin n'est pas vraiment une réponse à une question de cette forme, mais une réponse qui fait disparaître la question. Une telle idée a permis à Wittgenstein de faire sens de l'idée selon laquelle la clarté à laquelle aspire la philosophie est une clarté totale $e^{85}$.

La description que fait Hertz de la manière dont la nature problématique d'une notion supposément fondamentale de mécanique est expéri-

83. Sur la parenté entre le Tractatus et les Principes de Hertz, voir Barker, I980.

84. Hertz, I956, p. 7. (NDLT: ma traduction, d'après la version anglaise.)

85. Recherches philosophiques, $\mathbb{I}$ I33, p. 89. 
mentée (avec douleur, doute, embarras, inquiétude) tient également une part importante dans sa conception. Il mentionne l'expérience selon laquelle

il est extrêmement difficile d'exposer devant un auditoire attentif les rudiments de la mécanique, sans être parfois embarrassé, sans avoir de temps en temps l'envie de s'excuser, sans souhaiter en avoir fini le plus vite possible avec les rudiments, et pouvoir passer aux exemples qui parlent d'eux-mêmes ${ }^{86}$.

Une telle description a dû faire sur Wittgenstein une forte impression. Celui-ci s'est sûrement rappelé avoir vécu une expérience similaire dans nombre de cas alors qu'il était professeur de philosophie. Hertz entend évidemment signifier que nous devons prendre notre sensibilité conceptuelle au sérieux, même quand elle va contre l'autorité de la tradition.

Dans une situation idéale, quand nos expressions auront été reformulées de telle manière que les inquiétudes auront disparu et que nous ne serons plus enclins à poser des questions non légitimes, alors toutes choses seront laissées en l'état. Je considère que l'idée hertzienne exprimée dans le passage de Wittgenstein cité ci-dessus, d'une part, et l'une des remarques les plus cruciales que Wittgenstein ait fait quant à sa conception de la philosophie, d'autre part, sont intimement liées. La remarque que j'ai à l'esprit se trouve dans la section I24 des Recherches; Wittgenstein y dit que la philosophie, en fin de compte, laisse toutes choses en l'état, et il mentionne en particulier les mathématiques: "[La philosophie] laisse aussi les mathématiques en l'état, et aucune découverte mathématique ne peut la faire aller de l'avant ${ }^{87}$."

Selon la lecture que j'en fais, cette remarque constitue un défi lancé aux attitudes et aux attentes héritées de la philosophie traditionnelle quant à ce que la philosophie devrait accomplir «en fin de compte » : une doctrine substantielle et finale.

On pourrait affirmer que, dans sa philosophie, Wittgenstein a fait bien plus avec l'idée hertzienne de clarification conceptuelle que ce que Hertz a pu faire avec celle-ci dans sa physique.

Il est vain de rechercher la seule et unique source d'inspiration de Wittgenstein. Cependant, Hertz compte certainement au nombre des penseurs qui ont eu un énorme poids sur sa pensée philosophique. L'examen de l'influence de Hertz permet de tracer une ligne de continuité claire depuis le Tractatus jusqu'aux écrits tardifs de Wittgenstein.

Traduit de l'anglais par Anne-Marie Boisvert

86. Hertz, I956, p. 6-7. (NDLT: ma traduction, d'après la version anglaise.)

87. Recherches philosophiques, $\mathbb{S}$ I 24, p. 87 . 


\section{Bibliographie}

Baker, G. I988. Wittgenstein, Frege and the Vienna Circle, Oxford, Basil Blackwell.

- 2003. The voices of Wittgenstein: the Vienna Circle / Ludwig Wittgenstein and Friedrich Waismann; textes originaux allemands transcrits, édités et présentés par Gordon Baker. Traduction anglaise de Gordon Baker, Londres, Routledge.

Barker, P. I980. Hertz and Wittgenstein, Studies in History and Philosophy of Science 11, 243-256.

Benacerraf, P. et H. Putnam (dir.). I983. Philosophy of Mathematics, Selected Readings, deuxième édition, Cambridge, Cambridge U.P.

Corry, L. 2000. The Empiricist Roots of Hilbert's Axiomatic Approach, dans Proof Theory: History and Philosophical Significance, Vincent F. Henricks et al (eds), Dordrecht, Kluwer, p. 35-54.

- 2006a. "Axiomatics, Empiricism, and Anschauung in Hilbert's Conception of Geometry: Between Arithmetic and General Relativity », dans The Architecture of Modern Mathematics: Essays in History and Philosophy, J. Ferreirós et J. J. Gray (dir.) Oxford, Oxford University Press, I 55-I 76.

- 2006b. "The Origin of Hilbert's Axiomatic Method", dans The Genesis of General Relativity, Vol. 4. Theories of Gravitation in the Twilight of Classical Physics: The Promise of Mathematics and the Dream of a Unified Theory, J. Renn et al (dir.), New York, Springer, p. I39-236.

Detlefsen, M. I993a. "The Kantian Character of Hilbert's Formalism», dans J. Czermak (dir.), Proceedings of the $15^{\text {th }}$ International Wittgenstein-Symposium, Vienne, Verlag Hölder-Pichler-Temsky, p. I95-205.

- I993b. "Hilbert's Formalism ", dans Hilbert, Revue internationale de philosophie, 47, p. 285-304.

Einstein, A. I973. Ideas and Opinions, Londres, Souvenir Press.

Epple, M. 2003. "The End of the Science of Quantity: Foundations of Analysis, I 860-I9IO", dans Jahnke 2003, p. 29I-323.

Ewald, W. I996. From Kant to Hilbert. Readings in the Foundations of Mathematics, Oxford, Oxford University Press.

Floyd, J. 2000. "Wittgenstein, Mathematics and Philosophy", dans The New Wittgenstein, A. M. Crary et R. Read (dir.), Routledge, Londres, p. 232-26I.

Frege, G. I962. Grundgesetze der Arithmetik, vol. II, Georg Olms, Hildesheim, Verlagsbuchhandlung.

- I980. Philosophical and Mathematical Correspondence, B. McGuinness (dir.), Chicago, Univ. of Chicago Press.

Gerrard, S. I991. "Wittgenstein's Philosophies of Mathematics ", Synthese 87, p. $125-\mathrm{I} 42$.

Gray, J. J. 2006. "Modern Mathematics as a Cultural Phenomenon ", dans The Architecture of Modern Mathematics, J. Ferreirós et J. J. Gray (dir.), New York, Oxford University Press, 2006, p. 371-396.

Hertz, H. 1956. The Principles of Mechanics Presented in a New Form, New York, Dover. (Traduction anglaise de Die Prinzipen der Mechanik in neuem Zusammanhange dargestellt, Leipzig, I894.)

Hilbert, D. I902. Die Grundlagen der Geometrie, traduit en anglais sous le titre: The Foundations of Geometry, par E. J. Townsend, Chicago, The Open Court Publ. Co. 
-. I9I8. Axiomatisches Denken, Mathematische Annalen 78, p. 405-4I 5. Traduit en anglais sous le titre: "Axiomatic thought ", dans Ewald I996.

- I926. Über das Unendliche, Mathematische Annalen 95, p. I6I-I90. Traduit en anglais sous le titre: "On the infinite" dans P. Benacerraf et H. Putnam (dir.), 1983 .

- I930. Naturerkennen und Logik, in Hilbert: Gesammelte Adhandlungen, Dritter Band, (New York, Chelsea Publishing Company, I965). Traduit en anglais sous le titre: "Logic and the Knowledge of Nature", dans Ewald I996, p. II 57-II65.

Hilmy, S.S. 1987. The Later Wittgenstein, The Emergence of a New Philosophical Method, Oxford, Basil Blackwell.

Jahnke, H. N. I993. "Algebraic Analysis in Germany, I780-I 840: Some Mathematical and Philosophical Issues ", Historia Mathematica 20, $265-284$.

- (dir.), 2003. A History of Analysis, Providence RI, American Mathematical Society.

Kjaergaard, P.C. 2002. "'Hertz' and Wittgenstein's Philosophy of Science », Journal for General Philosophy of Science 33, I 2 I-I 49.

Mancosu, P. I998. From Brouwer to Hilbert, The Debate on the Foundations of Mathematics in the 1920s, Oxford, Oxford University Press.

McGuinness, B. 2005. "Wittgenstein: Philosophy and Literature", dans A. Pichler et S. Säätelä (dir.), Wittgenstein: The Philosopher and his Works, WAB, Bergen, p. 326-340.

- (dir.). I979. Wittgenstein and the Vienna Circle: Conversations Recorded by Friedrich Waismann, traduction anglaise de J. Schulte et B. McGuinness, Oxford, Blackwell.

McGinn, M. 2006. Elucidating the Tractatus, Wittgenstein's Early Philosophy of Logic \& Language, Oxford, Oxford University Press.

Medina, José. 2004. "Wittgenstein's Social Naturalism: The Idea of "Second Nature" After the Philosophical Investigations", dans The Third Wittgenstein, The Post-Investigations Works, D. Moyal-Sharrock (dir.), Ashgate, Hampshire.

Mehrtens, H. I990. Moderne - Sprache - Mathematik: Eine Geschichte des Streits um die Grundlagen der Disziplin und des Subjekts formaler Systeme, Francfort, Suhrkamp.

Monk, R. I990. Ludwig Wittgenstein, The Duty of Genius, The Free Press, New York, Macmillan.

Mülhölzer, F. 2005. "A Mathematical Proof Must be Surveyable - What Wittgenstein Meant by this and What it Implies ", Grazer Philosophische Studien 7I, p. 57-86.

- 2008. "Wittgenstein und der Formalismus", dans "Ein Netz von Normen": Wittgenstein und die Mathematik, M. Kroß (dir.), Berlin, Parerga Verlag, p. IO7-I 48 .

Van Heijenoort, J. (dir.). 1967. From Frege to Gödel: A Source-Book in Mathematical Logic, 1879-1931, Cambridge MA, Harvard Univ. Press.

Visser, H. I999. "Boltzmann and Wittgenstein on how Pictures Became Linguistic ", Synthese 119, I35-I 56.

Wilson, A.D. I989. "Hertz, Boltzmann and Wittgenstein Reconsidered », Studies in History and Philosophy of Science 20, 245-263. 


\section{Guvres de Wittgenstein utilisées par l'auteur de cet article:}

Wittgenstein, L. [TLP]: Tractatus Logico-Philosophicus. Traduction anglaise de D. F. Pears et B. F. McGuinness, Londres, Routledge \& Kegan Paul, I969.

- . [PR]: Philosophical Remarks, R. Rhees (dir.). Traduction anglaise de R. Hargreaves et R. White, Oxford, Blackwell, deuxième édition, 1975.

- [PG]: Philosophical grammar, R. Rhees, (dir.). Traduction anglaise de A. J. P. Kenny, Oxford, Blackwell, I974.

- $[\mathrm{BB}]$ : The Blue and Brown Books: Preliminary Studies for the 'Philosophical Investigations', R. Rhees (dir.), Oxford, Blackwell, I958.

- $[\mathrm{PI}]$ : Philosophical Investigations, G.E.M. Anscombe et R. Rhees (dir.). Traduction anglaise de G. E. M. Anscombe, Oxford, Blackwell, $2^{\text {nd }}$ dir., 1958.

- [LFM]: Wittgenstein's Lectures on the Foundations of Mathematics Cambridge, 1939, C. Diamond (dir.), Hassocks, Sussex, The Harvester Press, I976.

- [RFM]: Remarks on the Foundations of Mathematics, G.H. von Wright, R. Rhees et G. E. M. Anscombe (dir.). Traduction anglaise de G. E. M. Anscombe, Oxford, Blackwell, $3^{\text {rd }}$ dir., I978.

- [Z]: Zettel, G. E. M. Anscombe et G. H. von Wright (dir.). Traduction anglaise de G. E. M. Anscombe, Oxford, Blackwell, $2^{\text {nd }}$ dir., I98 I.

- [AL]: Wittgenstein's Lectures, Cambridge, 1932-1935: From the Notes of Alice Ambrose and Margaret Macdonald, Alice Ambrose (dir.), Chicago, University of Chicago Press, I979.

- [DL]: Wittgenstein's lectures, Cambridge 1930-1932, from the notes of J. King and D. Lee, Desmond Lee (dir.), Oxford, Blackwell, I980.

. [CV]: Culture and Value (dir.). G. H. von Wright. Traduction anglaise de Peter Winch, Oxford, Blackwell, I998.

- [WVC]: Ludvig Wittgenstein and the Wienna Circle: Conversations Recorded by Friedrich Waismann. B. F. McGuiness (dir.). Traduction anglaise de J. Schulte et B. McGuiness, Oxford, Blackwell, I979.

- [RPP I]: Remarks on The Philosophy of Psychology, vol. I, G. E. M. Anscombe et G. H. von Wright (dir.). Traduction anglaise de G. E. M. Anscombe, Oxford, Blackwell, I980.

- [PPO]: Ludwig Wittgenstein, Public and Private Occasions, J. C. Klagge et A. Nordmann (dir.), Oxford, Rowman \& Littlefield Publishers, 2003.

\section{Fuvres de Wittgenstein utilisées dans la traduction française de cet article:}

Wittgenstein, L. Carnets 1914-1916. Traduction française de G.-G. Granger, Paris, Gallimard, I97I.

—. Cours sur les fondements des mathématiques. Cambridge 1939. Traduction française avec textes anglais en regard par É. Rigal, Mauvezin, TER, I 992.

—. De la certitude. Traduction française de J. Fauve, Paris, Gallimard, I 976.

—. Dictées de Wittgenstein à Waismann et pour Schlick. Textes inédits (années I930), sous la direction de A. Soulez, textes établis par G. Baker et traduits par C. Chauviré et al, Paris, PUF, I996.

—. Fiches. Traduction française de J.-P. Cometti et É. Rigal, Paris, Gallimard, 2008. 
- Grammaire philosophique. Traduction française de M.-A. Lescourret, Paris, Gallimard, I980.

- Le cahier bleu et le cahier brun, traduction française de G. Durand, Paris, Gallimard, I965.

- Les cours de Cambridge 1930-32, établis par D. Lee (à partir des notes de J. King et de D. Lee). Traduction française avec textes anglais en regard par É. Rigal, Mauvezin, TER, I988.

- Les cours de Cambridge 1932-35, établis par A. Ambrose (à partir des notes d'A. Ambrose et de M. Macdonald). Traduction française avec textes anglais en regard par É. Rigal, Mauvezin, TER, I992.

- Recherches philosophiques, traduction française de F. Dastur et al, Paris, Gallimard, 2004.

- Remarques mêlées, éditées par G. H. von Wright et H. Nyman. Traduction française avec le texte allemand en regard par G. Granel, Mauvezin, TER, I990.

—. Remarques philosophiques, éditées par R. Rhees. Traduction française de J. Fauve, Paris, Gallimard, I975.

- Remarques sur les fondements des mathématiques, éditées par G. E. M. Anscombe, R. Rhees et G. H. von Wright. Traduction française de M.-A. Lescourret, Paris, Gallimard, I983.

—. Tractatus Logico-Philosophicus. Traduction française de G.-G. Granger, Paris, Gallimard, I993.

Wittgenstein et le Cercle de Vienne (I929-32), établis par B. McGuinness d'après les notes de F. Waismann. Traduction française avec texte allemand en regard par G. Granel, Mauvezin, TER, I99I.

\section{Autre ouvrage cité dans la traduction française de cet article:}

F. Rivenc et P. de Rouilhan (dir.). Logique et fondements des mathématiques. Anthologie (1850-1914), Paris, Payot, I992. 